\title{
Flow around an inclined circular disk
}

\author{
SONG GAO ${ }^{1,2, \S}$, LONGBIN TAO ${ }^{3, \S}$, XINLIANG TIAN $^{1,2} \dagger$, \\ JIANMIN YANG ${ }^{1,2}$ \\ ${ }^{1}$ State Key Laboratory of Ocean Engineering, Shanghai Jiao Tong University, Shanghai \\ 200240, China \\ ${ }^{2}$ Collaborative Innovation Center for Advanced Ship and Deep-Sea Exploration, Shanghai \\ 200240, China \\ ${ }^{3}$ Department of Naval Architecture, Ocean \& Marine Engineering, University of Strathclyde, \\ Glasgow, G4 0LZ, UK
}

(Received ?; revised ?; accepted ?. - To be entered by editorial office)

Direct numerical simulations are performed for the uniform flow around an inclined circular disk. The diameter-thickness aspect ratio $\left(\chi=D / t_{d}\right)$ of the disk is 50 and the inclination angle $(\alpha)$ is considered over the range of $0^{\circ} \leqslant \alpha \leqslant 80^{\circ}$, where $\alpha=0^{\circ}$ refers to the condition where the flow is normal to the disk. The Reynolds number $(R e)$, based on the short axis of projection in the streamwise direction, is defined as $R e=U_{\infty} D \cos \alpha / \nu$, where $U_{\infty}$ is the velocity of the flow and $\nu$ is the kinematic viscosity. Re is investigated over the range of $50 \leqslant R e \leqslant 300$. In the considered $R e-\alpha$ parametric space, five states are observed and denoted as: (I) steady state (SS); (II) periodic state (PS); (III) periodic state with a low frequency modulation (PSL); (IV) quasi-periodic state (QP), and (V) chaotic state (CS). Both Re and $\alpha$ affect the bifurcation mechanism. The bifurcating sequence occurring at $\alpha=0^{\circ}$ is generally observed over the whole $R e-\alpha$ space, although it is advanced at small $\alpha$ and delayed at large $\alpha$. The advancement of thresholds for different states is due to the effects introduced by inclination, which tend to select the plane of symmetry for the wake in order to regulate the wake and intensify some flow features. Nevertheless, the bifurcations are still in the dominant position when leading a state without stable symmetry, i.e. the planar symmetry could not be recovered by small $\alpha$. These phenomena are further discussed with respect to the vortex shedding patterns behind disk. Furthermore, for any fixed disk, the wake behaviour is only associated with that found in the steady vertical state of freely falling disk. The fully coupled fluid-body system is fundamentally different from the fixed cases.

Key words: flow-structure interactions, bifurcation, vortex instability, transition, wakes

\section{Introduction}

Flow past two prototypical axisymmetric geometries, spheres and circular disks, have been extensively investigated in the literatures. It is well known that the wake of these simple bodies experiences complicated transition scenarios from steady state to chaotic state with a corresponding increase in the Reynolds number. For a sphere, a consensus on its transition scenarios has been obtained via extensive numerical and experimental investigations (see, e.g. Johnson \& Patel 1999; Natarajan \& Acrivos 1993; Tomboulides

$\dagger$ Email address for correspondence: tianxinliang@sjtu.edu.cn. §These authors contributed equally to this work. 
\& Orszag 2000). In the case of the same problem for a circular disk, although some similar flow characteristics are presented (Fabre et al. 2008), significant variability in the results has been reported. Some experimental studies (see, e.g. Marshall \& Stanton 1931; Willmarth et al. 1964; Kuo \& Baldwin 1967; Roos \& Willmarth 1971; Roberts 1973; Berger et al. 1990; Fernandes et al. 2007; Zhong \& Lee 2012) and numerical studies (see, e.g. Michael 1966; Rimon 1969; Rivet et al. 1988; Shenoy \& Kleinstreuer 2008; Fabre et al. 2008; Auguste et al. 2010; Chrust et al. 2010; Shenoy \& Kleinstreuer 2010; Yang et al. 2014a 2015) have investigated the case of a circular disk. The transition of the wake behind a fixed circular disk normal to the uniform flow is determined by two key parameters, the Reynolds number $(R e)$ and the aspect ratio $(\chi)$ of the disk. According to the previous results, the transition scenarios around circular disks involve several stages as follows.

When the Reynolds number is sufficiently low, the flow is in an axisymmetric and steady state (Shenoy \& Kleinstreuer 2008). The first bifurcation is observed for all considered disks, e.g. a flat disk (corresponding to an infinitely thin disk of $\chi=\infty$ ) investigated by Natarajan \& Acrivos (1993); Fabre et al. (2008) and Meliga et al. (2009) and disks of non-zero thickness, considered by Fernandes et al. (2007) $(\chi=2,3,4,6$ and 10), Shenoy \& Kleinstreuer (2008) $(\chi=10)$, and Auguste et al. $(2010)(\chi=3)$. The first bifurcation is regular and occurs at a critical Reynolds number of $R e_{c 1}$, giving rise to a steady flow of reflectional symmetry. The breaking of axisymmetry results in a pair of steady streamwise vortices and a steady lift force in the symmetric plane which has an arbitrary orientation determined by the initial conditions (Chrust et al. 2010). This state is called the 'steady state' (SS) (Fabre et al. 2008; Meliga et al. 2009) or 'steady asymmetric' state (Shenoy \& Kleinstreuer 2008). The value of $R e_{c 1}$ is a monotonic decreasing function of $\chi$ as $1+\chi^{-1}$ (Fernandes et al. 2007). For flat disks $(\chi=\infty)$, a reasonable consensus has been reached for the value of $R e_{c 1}$ between 115 and 117 (see, e.g. Chrust et al. 2010; Fabre et al. 2008; Meliga et al. 2009; Natarajan \& Acrivos 1993). For a disk of $\chi=10, R e_{c 1}$ is found to be 135 (Shenoy \& Kleinstreuer 2008) and 129.6 (Chrust et al. 2010). For a thicker disk of $\chi=3, R e_{c 1}$ is reported to be approximately 159.4 .

While a steady state with planar symmetry is common to all of these cases, for the second bifurcation, which is always of Hopf type, the transition scenarios are very different. For a disk of $\chi=10$, the secondary Hopf bifurcation leads to a state featured by 'three-dimensional periodical flow with regular rotation' (Shenoy \& Kleinstreuer 2008). The periodic lift force oscillates around a non-zero mean value and the oscillations are perpendicular to the plane selected by the primary bifurcation, resulting in a loss of reflectional symmetry. This state is also referred to as 'reflectional symmetry breaking' (RSB) (Fabre et al. 2008), 'mixed mode with phase $\pi$ ' $\left(\mathrm{MM}_{\pi}\right)$ (Meliga et al. 2009), and 'Yin-Yang' (YY) (Auguste et al. 2010). The critical Reynolds number for the Hopf bifurcation, $R e_{c 2}$, is a fuction of $1+\chi^{-1}$ as well (Fernandes et al. 2007). $R e_{c 2}$ has been reported to be between 121 and 125.6 for a flat disk (see, e.g. Chrust et al. 2010; Fabre et al. 2008; Meliga et al. 2009; Natarajan \& Acrivos 1993), and between 136.3 and 138.7 (Chrust et al. 2010) or approximately 155 (Shenoy \& Kleinstreuer 2008) for $\chi=10$. The transition scenario of a flat disk, in which the RSB mode remains as the secondary bifurcation, holds up by the thin bodies with $\chi \geqslant 4$ (Chrust et al. 2010). Alternatively, for a thicker disk with $\chi=3$, 'Yin-Yang' state is the fourth bifurcation because two exclusive bifurcations, namely 'zig-zig' and 'knit-knot' modes, are encountered at the second and third thresholds respectively (Auguste et al. 2010). The 'zig-zig' mode is characterised by reflectional symmetry with a periodic lift force oscillating around a non-zero mean, equal 
to 'reflectional symmetry preserving' (RSP) mode. The 'knit-knot' mode is characterised by net non-zero helicity.

As the Reynolds number increases further, the amplitude of the oscillations in the RSB mode increases and the non-zero mean value recesses gradually until reflectional symmetry is recovered. This state is referred to as the 'standing wave' (SW) (see, e.g. Fabre et al. 2008; Meliga et al. 2009), 'unsteady with planar symmetry and zero lift force' (Shenoy \& Kleinstreuer 2008) or 'zig-zag' (Auguste et al. 2010). The threshold of this third bifurcation for a flat disk, $R e_{c 3}$, is found to be approximately 140 (Fabre et al. 2008), 143 (Meliga et al. 2009), and between 142 and 143 (Chrust et al. 2010). In the case of a disk with $\chi=3$, the critical Reynolds number of this fifth bifurcation is found to be around 215 (Auguste et al. 2010).

Before the flow eventually develops into a fully chaotic state, a quasi-periodic (or pre-chaotic) mode is formed, which is characterised by the persistence of a reflectional symmetry plane and emergence of a secondary frequency close to one-third of the leading frequency of the previous regimes (Auguste et al. 2010; Chrust et al. 2010). Unlike other thresholds that can be determined precisely, the exact limit between quasi-periodicity and chaos is difficult to determine.

A comprehensive $R e-\chi$ parametric study of the transition scenarios of circular disks and oblate spheroids have been conducted (Chrust et al. 2010). It is reported that a thin oblate spheroid $(\chi>10)$ also presents a flat-disk-like scenario so that it is reasonable to believe the physics of flow around thin bodies at low Reynolds number are similar, independently of their geometries.

The fluid dynamics of a fixed circular disk has been exhaustively presented in the literatures. Nevertheless, freely moving bodies are more common in realistic scenarios, e.g. falling coins in water and dead leaves in air. Extensive investigations have been conducted on the wake instabilities and paths of a freely falling (or rising) disk (see, e.g. Willmarth et al. 1964; Field et al. 1997; Zhong \& Lee 2012; Auguste et al. 2013; Chrust et al. 2013) and spheres or oblate spheroids (see, e.g. Jenny et al. 2004; Horowitz \& Williamson 2010; Zhou et al. 2017). For disks, this problem depends on three control parameters, the disk aspect ratio $\chi$, the body-to-fluid ratio $\bar{\rho}$ (also denoted as the dimensionless moment inertia $I^{*}$, see, e.g. Willmarth et al. 1964; Field et al. 1997; Zhong \& Lee 2012), and the Archimedes number $A r$ (Reynolds number based on gravitational velocity, which is also defined as the Galileo number $G$, see, e.g. Chrust et al. 2013). Hence, for a disk of given aspect ratio, inertial and viscous effects together control the transitional process. A preliminary transition map of a freely falling thin circular disk is given by Field et al. (1997). When the Reynolds number is low, i.e. viscous effects are significant, the motion of the falling disk is found to be steady and vertical, also denoted as the 'straight vertical' (SV) mode (Auguste et al. 2013). At higher Reynolds numbers, a periodic fluttering state, corresponding to the 'zig-zag' (ZZ) mode, is observed for disks of small $\bar{\rho}$, while a tumbling state, i.e. 'autorotation' (AR), is found for large $\bar{\rho}$. Between these states is an intermediate chaotic state, characterised by a random switching between $\mathrm{ZZ}$ and AR modes. The paths in these states are all of planar symmetry. More recently, some regimes with small path deviations between $\mathrm{SV}$ and $\mathrm{ZZ}$ are reported (Auguste et al. 2013) along with other regimes with three-dimensional trajectories (Zhong et al. 2011). Further information could be found in a detailed review (Ern et al. 2012). Regretfully, connections between wake instability of a fixed disk normal to flow and that of freely falling one are limited (Fernandes et al. 2007; Auguste et al. 2013). However, a freely moving disk is usually exposed to the flow at an inclined state, which leads us to expect the study on flow around a fixed inclined disk might help us better understand its physics.

Figure 1 shows a schematic diagram of the uniform flow around a fixed inclined disk. 


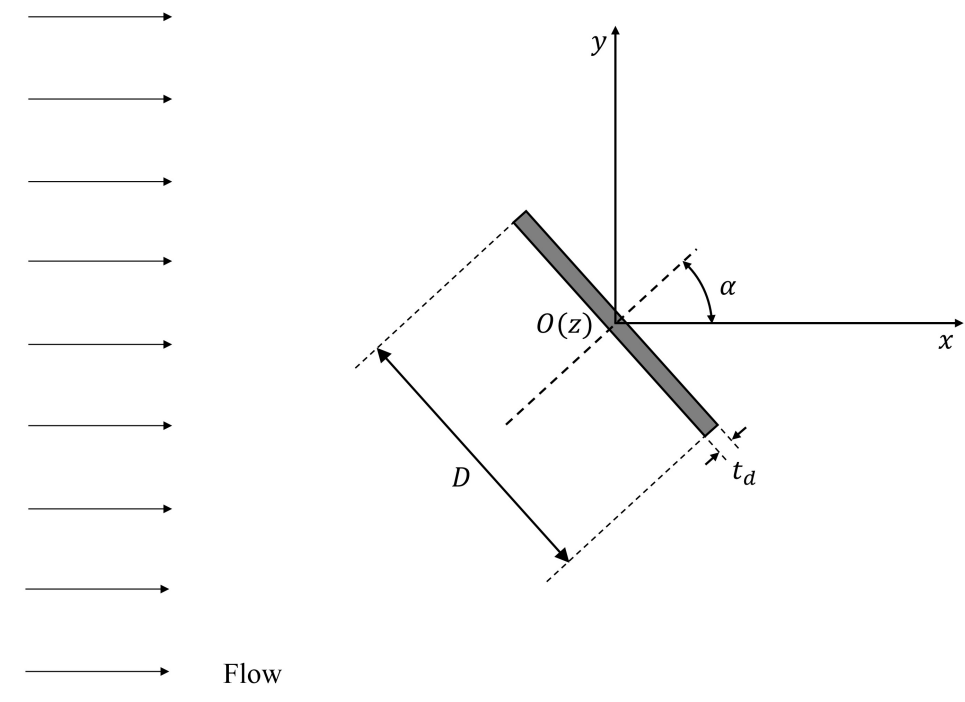

FIGURE 1. Schematic representation of the uniform flow past an inclined circular disk. The disk parameters and $\alpha$ are demonstrated. The coordinate system is defined as well, where the direction of $x$-axis coincides with the direction of the incoming flow.

The pioneer work of this topic is the experimental study by Calvert (1967), in which the results are obtained from smoke visualization experiments in an open-return wind tunnel. During the experiments, $\alpha$ is up to $50^{\circ}$, and $R e$ is in the range of 3500 to 5000 . Vortex shedding of increasing frequency with increasing inclination angle is observed. Recently, Chrust et al. (2015) conducted both numerical and experimental studies of the flow past an inclined disk of $\chi=6$ and $\infty$. The $R e$ is up to 250 and $\alpha$ reached $60^{\circ}$. The effects of the inclination on the wake transition, mainly at Hopf bifurcation, are discussed considering both extremely small inclinations $\left(\alpha<2^{\circ}\right)$ and large inclinations $\left(\alpha>4^{\circ}\right)$. A relatively poor agreement between vortex shedding frequencies is observed for numerical and experimental results, which is attributed to the confinement of the experiments and the methods for supporting the disks. Tian et al. (2017a) conducted a direct numerical simulation (DNS) study of the flow behind an inclined disk of $\chi=50$. At $R e=500$ (disk diameter based), the disk is inclined by six inclination angles over the range of $\left[0^{\circ}, 60^{\circ}\right]$ and four types of flow patterns are categorized according to the lift coefficients, pressure coefficients, three-dimensional vortical structures, etc. In aerodynamics, flow past low-aspect-ratio wings with some angles of attack at low Reynolds number has been investigated to explore the lift experienced by geometries or the rich dynamics provided by separated flow (Dickinson \& Gotz 1993; Dong et al. 2006; Taira \& Colonius 2009). Compared with normal rectangular wings, some semicircular/ elliptical wings which have curved leading edge appear to give rise to gentler wake under the same flow configuration. Surprisingly, these foils experience similar lift force which is most likely due to the viscous nature of the flows at low Reynolds number (Taira \& Colonius 2009).

Even though extensive research regarding disks has been performed to date, several factors still require further elucidation. For example: (i) what is the wake transition behind an inclined disk? (ii) how many types of flow regimes exist? (iii) how do these regimes distribute and where are the thresholds between them? (iv) how does inclination influence the wake of a circular disk? (v) are there any connections between fixed inclined disks and freely moving disks? Therefore, it is necessary to conduct a comprehensive 
parametric study in the $R e-\alpha$ space, which is expected to provide answers to these questions.

Driven by these motivations, DNS investigations are performed to reveal the fundamental physical issues behind this problem. In such studies, numerical methods are preferable to experimental ones, as ideal conditions (e.g. precise inclination angles and perfect flow conditions) can be more easily achieved and problems with supporting structures for the disk could be avoided. In the present study, the range of $R e$ is considered as 50 to 300 since it is wide enough for a thin disk at $\alpha=0^{\circ}$ experiencing the whole process from steady to chaotic state. Moreover, in our preliminary tests, when $\alpha$ increases to $80^{\circ}$ at $R e=300$, flow behind disk experiences another transitional process from chaotic to steady state and $\alpha=80^{\circ}$ is high enough for flow remaining steady on the whole range [0, $300]$ in $R e$. Consequently, the ranges of $R e$ and $\alpha$ are confirmed. In this proposed $R e-\alpha$ domain, all the main bifurcations for low Reynolds number flow around inclined disk are expected to be found. Even though an infinitely thin disk is considered appropriate for fundamental theoretical studies, the disk with $\chi=50$ is a more realistic situation worthy of discussion and allows the possibility of the results being experimentally validated in the future.

The remainder of the paper is organized as follows. Details of numerical simulations are described in $\S 2$. The results are presented in $\S 3$ with conclusion and discussion being provided in $\S 4$.

\section{Numerical simulations}

\subsection{Mathematical formulations and numerical methods}

We consider a fixed circular disk, where its axis is inclined by $\alpha$ with respect to the uniform flow direction. The flow of an unsteady incompressible fluid is governed by the Navier-Stokes (N-S) equations, which are solved here in the Cartesian coordinate system $(x, y, z)$. These coordinates could be uniformly denoted as $x_{i}$, where $i=1,2,3$ and $u_{i}$ is the velocity component of the corresponding direction. The N-S equations are expressed as:

$$
\begin{gathered}
\frac{\partial u_{i}}{\partial x_{i}}=0 \\
\frac{\partial u_{i}}{\partial t}+u_{j} \frac{\partial u_{i}}{\partial x_{j}}=-\frac{1}{\rho} \frac{\partial p}{\partial x_{i}}+\nu \frac{\partial^{2} u_{i}}{\partial x_{j} \partial x_{j}}
\end{gathered}
$$

where $j=1,2,3, p$ is the pressure and $\rho$ is the density of the fluid. For clarity, the velocity components $u_{1}, u_{2}$, and $u_{3}$ are also denoted by $u_{x}, u_{y}$, and $u_{z}$, respectively.

These equations are discretized using the finite volume method (FVM) based on the open source computational fluid dynamics (CFD) code OpenFOAM. OpenFOAM is an object-oriented code, which enabled the operation and manipulation of tensorial data to solve continuum mechanics problems (Weller et al. 1998). The pressure implicit with splitting of operators (PISO) scheme (pisoFoam) is used in our numerical simulations. The discretization of each term is undertaken by integrating the term over a control volume using Gausss theorem, and then volume and surface integrals are linearized using suitable schemes. The spatial schemes of interpolation, gradient, Laplacian, and divergence are all linear and of second order. An additional correction is performed for the Laplacian term by interpolating cell centre gradients. The second-order CrankNicholson scheme is introduced for the time integration. Further detailed information of 

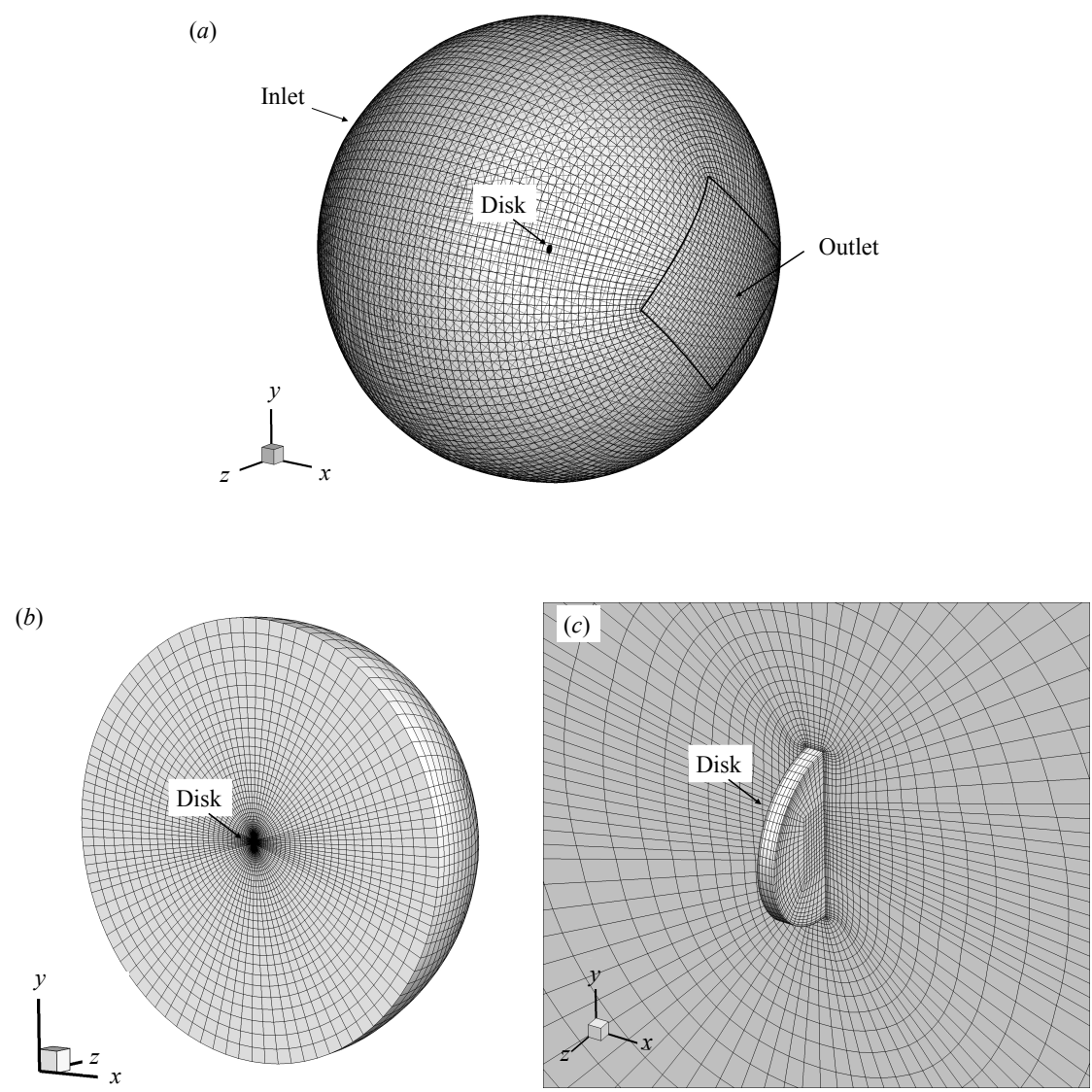

FIGURE 2. Fixed inclined disk: schematic diagrams of the computational domain and grid of the mesh. (a) Overall view of the spherical computational domain and the boundaries. The outlet boundary is located at the downstream of the disk and the inlet boundary covers the rest of the surface. (b) View of the grid structures in the plane $z / D=0$. (c) A detailed view of the grids near the disk surface.

these schemes is presented previously OpenFOAM (2009). The present code has been validated by our previous simulations (see, e.g. Tian et al. 2017a,b; Yang et al. 2014b).

As shown in figure 2(a), a spherical computational domain is applied for the numerical simulations. The origin of the Cartesian coordinate system $(x, y, z)$ is located at the centre of the disk, which is also the centre of the computational domain. The radius of the computational domain is denoted as $R_{d}$. The boundary conditions on the disk surface are no-slip and impermeability for the velocity and a zero normal gradient for the pressure. At the inlet boundary, a uniform flow $\left(U_{\infty}, 0,0\right)$ and a zero normal gradient pressure are set. At the outlet boundary, the velocity is set to a zero normal gradient and the pressure is set to zero. The whole computational domain is discretized with hexahedral elements, and the nonuniform grid distribution is used. Mesh is refined near the edge of the disk to better resolve the flow around it. Such method has been widely applied and verified in the literatures (Shenoy \& Kleinstreuer 2008; Auguste et al. 2010; Chrust et al. 2015). In order to simulate the flow past the disk inclined with different $\alpha$, the entire domain is rotated with the disk together by the same angle. Thus, a good mesh quality could be achieved conveniently for all simulations. 


\begin{tabular}{|c|c|c|c|c|c|c|c|c|c|c|}
\hline & Case & Elements & $n_{1} / D$ & $R_{d}$ & $\Delta t U_{\infty} / D$ & $(130,45)$ & $(175,35)$ & $(240,30)$ & $(250,60)$ & $(300,15)$ \\
\hline \multirow{4}{*}{$\left\langle C_{x}\right\rangle$} & A & 783,480 & 0.0030 & $30 \mathrm{D}$ & 0.0025 & 0.823 & 0.993 & 1.029 & 0.480 & 1.106 \\
\hline & B & $1,049,600$ & 0.0027 & $30 \mathrm{D}$ & 0.0020 & 0.822 & 0.993 & 1.034 & 0.480 & 1.127 \\
\hline & $\mathrm{C}$ & $1,347,552$ & 0.0025 & $30 \mathrm{D}$ & 0.0015 & 0.821 & 0.992 & 1.036 & 0.479 & 1.126 \\
\hline & D & 942,080 & 0.0027 & $20 \mathrm{D}$ & 0.0020 & 0.822 & 0.994 & 1.034 & 0.480 & 1.120 \\
\hline \multirow{4}{*}{$\left\langle C_{y}\right\rangle$} & A & 783,480 & 0.0030 & $30 \mathrm{D}$ & 0.0025 & 0.673 & 0.612 & 0.540 & 0.655 & 0.280 \\
\hline & B & $1,049,600$ & 0.0027 & $30 \mathrm{D}$ & 0.0020 & 0.673 & 0.613 & 0.544 & 0.655 & 0.284 \\
\hline & $\mathrm{C}$ & $1,347,552$ & 0.0025 & $30 \mathrm{D}$ & 0.0015 & 0.670 & 0.609 & 0.545 & 0.653 & 0.282 \\
\hline & D & 942,080 & 0.0027 & $20 \mathrm{D}$ & 0.0020 & 0.672 & 0.614 & 0.544 & 0.655 & 0.282 \\
\hline
\end{tabular}

TABLE 1. Mean values of the force coefficients $\left\langle C_{x}\right\rangle$ and $\left\langle C_{y}\right\rangle$ of five flow configurations (Re, $\alpha$ ) obtained via four cases with different spatial and temporal resolutions. $n_{1}$ is the size of the smallest cells near the disk surface and $\Delta t$ is the time step.

The non-dimensional force coefficients in three directions are defined as follows:

$$
\left(C_{x}, C_{y}, C_{z}\right)=\frac{\left(F_{x}, F_{y}, F_{z}\right)}{\frac{1}{8} \rho U_{\infty}^{2} \pi D^{2}}
$$

where $F_{x}, F_{y}$, and $F_{z}$ are the force components imposed on the disk in the $x, y$, and $z$ directions, respectively, which are directly calculated by integrating the pressure and viscous shear stress over the disk surfaces.

For the sake of the coherence with the definition of $R e$, the Strouhal number is nondimensionalized by the short axis of projection in the streamwise direction as follows:

where $f$ is the frequency.

$$
S t=\frac{f D \cos \alpha}{U_{\infty}}
$$

The vorticity component in the streamwise direction is defined as:

$$
\omega_{x}=\frac{\partial u_{z}}{\partial y}-\frac{\partial u_{y}}{\partial z}
$$

\subsection{Convergence studies}

Convergence studies for evaluating the effects of spatial and time resolutions on numerical simulations are performed for three cases with different number of grid elements and time steps, i.e. cases A, B, C in table 1. In each case, five typical flow configurations distributed evenly in the considered space are chosen for the comparison. $(R e, \alpha)=(130$, $\left.45^{\circ}\right)$ and $\left(240,30^{\circ}\right)$ are on or near thresholds; $\left(175,35^{\circ}\right)$ and $\left(250,60^{\circ}\right)$ are in the middle of map; $\left(300,15^{\circ}\right)$ is in the turbulent regime. One should note that for all the flow configurations, the results of $\left\langle C_{x}\right\rangle$ and $\left\langle C_{y}\right\rangle$ from these cases shows good agreement, indicating a good convergence for the spatial and temporal resolutions. The effects of the size of the computational domain on the numerical results are evaluated using a smaller spherical domain with $R_{d}=20 D$ (case D in table 1 ). Case D has a similar spatial resolution and the same time step as in case B. The results obtained from case D agree well with those from cases B and C, indicating that a spherical computational domain of $R_{d}=20 D$ is large enough to eliminate the influence of the outer boundary. Moreover, the time traces of $u_{x} / U_{\infty}$ for these flow configurations are shown in figure 3 . Very good agreement is obtained from the results of cases A, B, C, and D again. For 

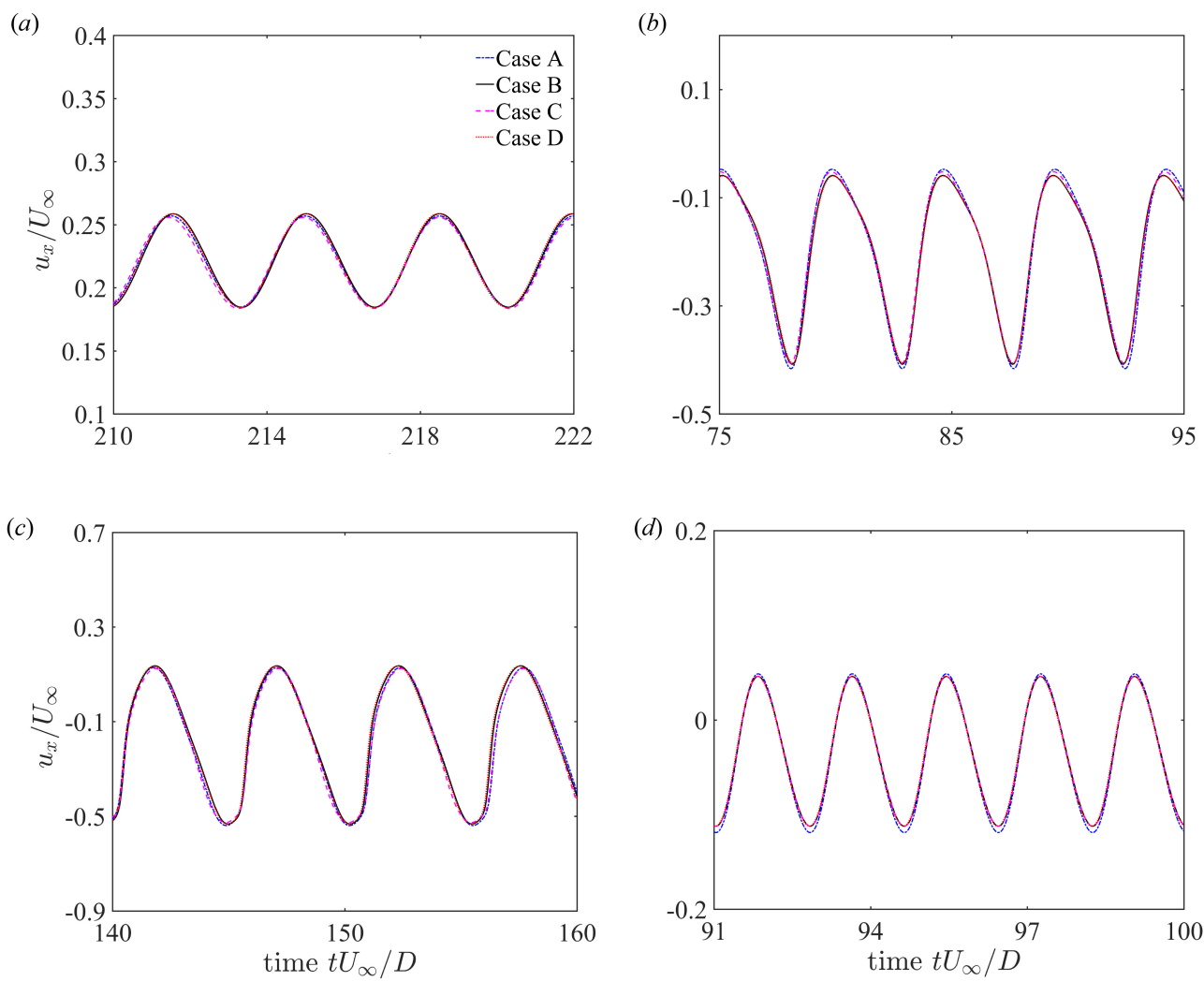

Figure 3. Comparison of the time traces of dimensionless axial velocity $u_{x} / U_{\infty}$ at the point of $x=1, y=z=0$ by four cases at $(\operatorname{Re}, \alpha)=(a)\left(130,45^{\circ}\right) ;(b)\left(175,35^{\circ}\right) ;(c)\left(240,30^{\circ}\right)$ and $(d)$ $\left(250,60^{\circ}\right)$. When the disk is inclined by $\alpha=15^{\circ}$ at $R e=300$, the flow shows a chaotic state; hence, the time traces could not be shown for comparison.

all the numerical simulations reported in the following sections, the conditions for case B, i.e. $1,049,600$ grid elements, a time step of $\Delta t U_{\infty} / D=0.002$, and a computational domain of $R_{d}=30 D$ are used.

\subsection{Code validation}

To examine the validity of our numerical method, the results of a well-documented flow configuration, i.e. a circular disk normal to the flow, are presented here and compared with results presented in the literatures. For a circular disk of $\chi=50$, the transition scenarios shows a total of five bifurcations from a steady flow to a completely chaotic state. Figure 4 demonstrates representative images of these states. Figure $4(a)$ shows the axial vorticity iso-surfaces after the primary bifurcation. The double-threaded wake illustrates that the flow is in a steady and reflectional symmetry state (c.f. figure 4 in Shenoy \& Kleinstreuer 2008). Note that the symmetry plane, which is arbitrarily oriented at approximately $72^{\circ}$ to the $y$-axis, is observed in our simulation. After the Hopf bifurcation, the loss of the symmetry plane (RSB mode) is indicated by the totally three-dimensional axial vorticity iso-surfaces (see figure $4 b$ ). The orientation of the lift force oscillates around $72.6^{\circ}$, which is the same plane selected as the primary bifurcation. The $C_{z} C_{y}$ diagram also shows the lift force oscillating along a closed path about a mean direction (c.f. figure 4 in Auguste et al. 2010). At the third bifurcation, as shown in figure 4(c), the reflectional 
(b)

(a)
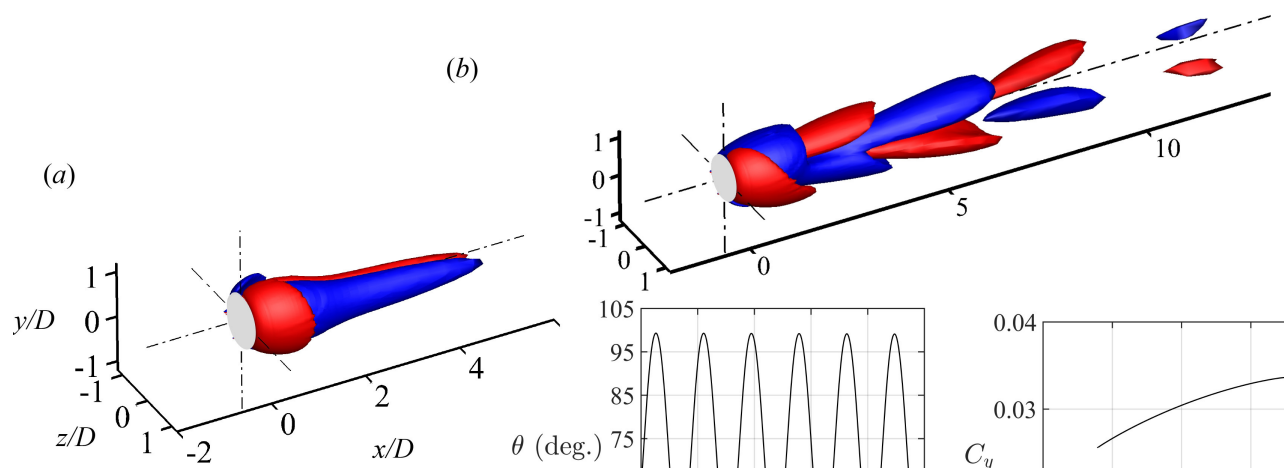

(c)
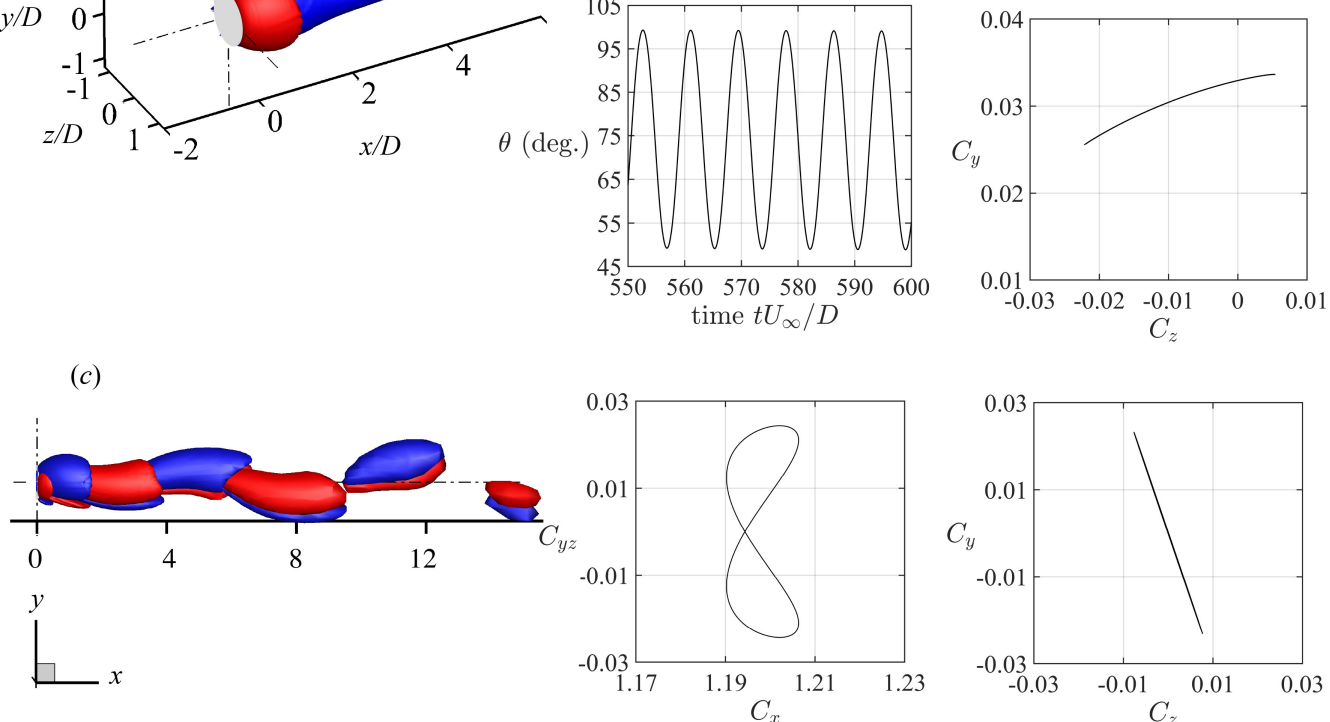

$(d)$
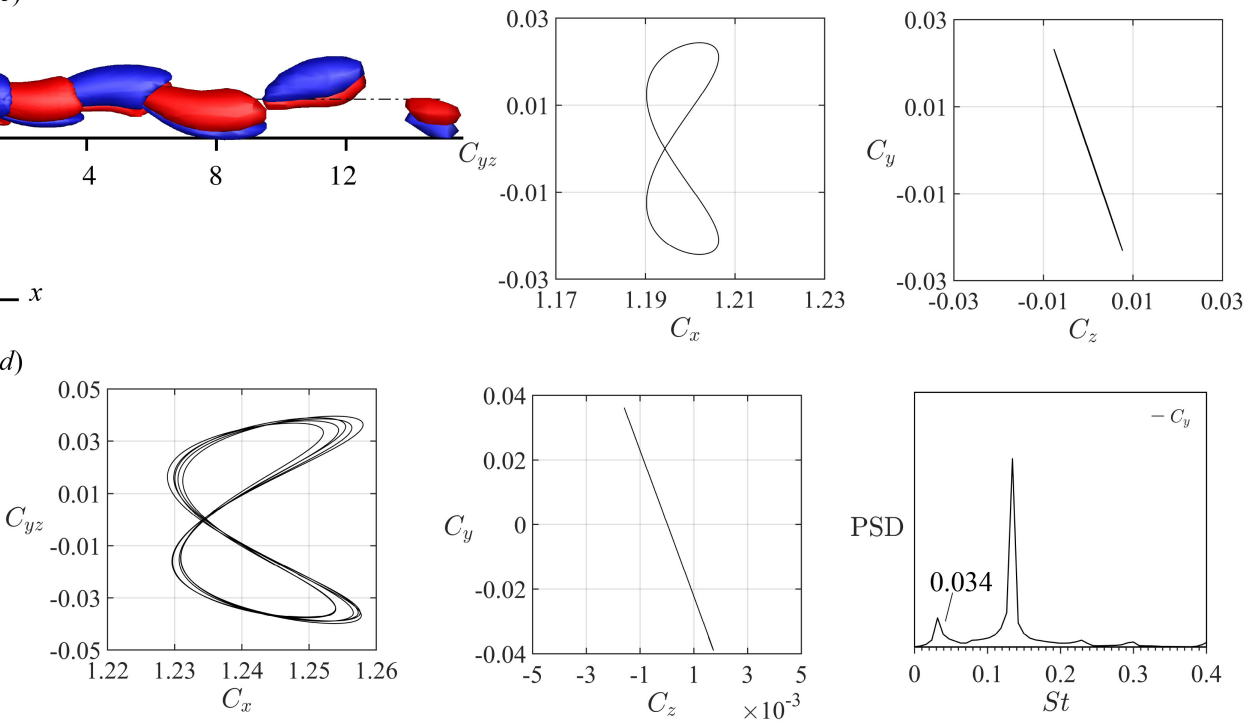

$(e)$
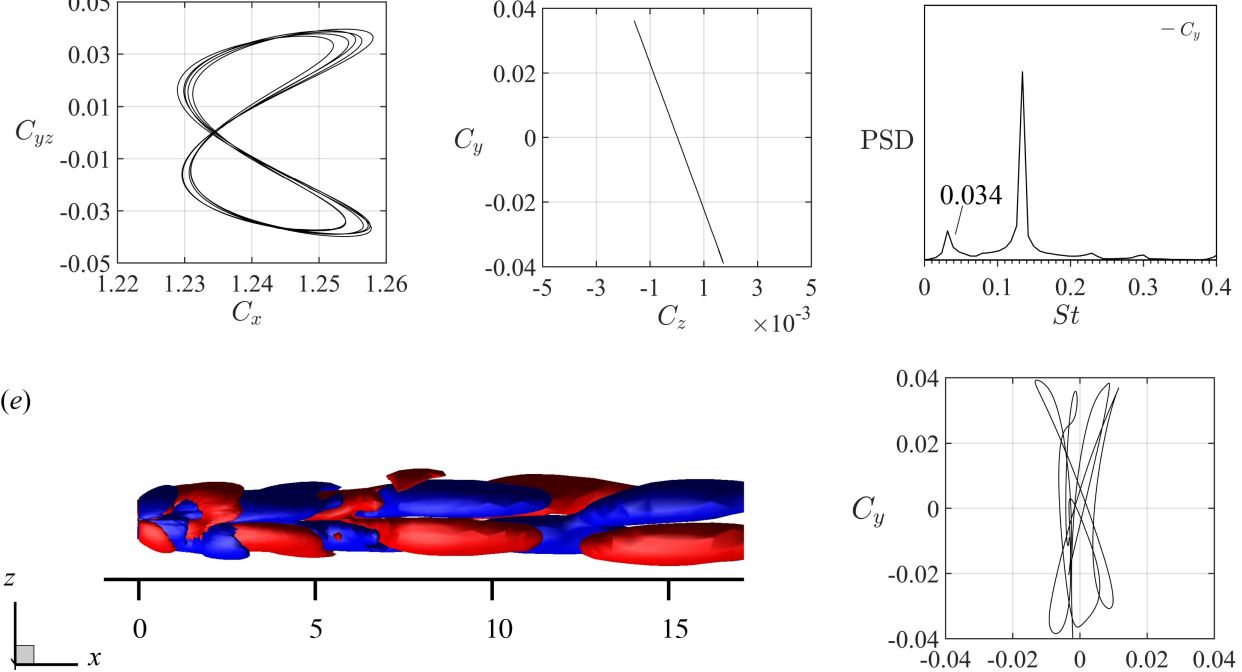

e)

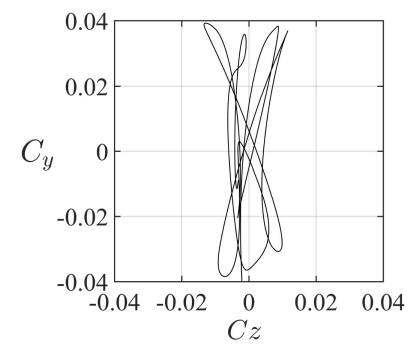

FiguRE 4. Sample characterisation of wake transition scenarios of a thin circular disk $(\chi=50)$ placed normal to the flow. $(a)$ steady and reflectional symmetry (after the primary regular bifurcation), $R e=125$. (b) periodic without reflectional symmetry or RSB (after the Hopf bifurcation), $R e=130$. The lift force oscillates around the plane selected by the primary bifurcation. $\theta$ is defined by the direction of the lift force with respect to the $y$-axis. $(c)$ periodic with recovered reflectional symmetry, $R e=141.5 . C_{y z}$ is the lift force coefficient. $(d)$ quasi-periodic, $R e=210$. A low frequency of $S t_{l}=0.034$ is observed. (e) chaotic state, $R e=220$. The three dimensional plots represent the iso-surfaces of a pair of equal magnitudes of positive and negative axial vorticity $\omega_{x}\left( \pm 0.1 U_{\infty} / D\right)$, indicated by the red and blue surfaces, respectively. The force coefficients for all parametric diagrams in the present study are plotted by more than ten whole periods. 


\begin{tabular}{|c|c|c|c|c|c|}
\hline$\chi$ & $R e_{c 1}$ & $\begin{array}{l}R e_{c 2} \\
S t_{2}\end{array}$ & $\begin{array}{l}\mathrm{Re}_{\mathrm{c3}} \\
\mathrm{St}_{3}\end{array}$ & $R e_{c 4}$ & $R e_{c 5}$ \\
\hline$\infty($ Fabre et al. 2008$)$ & $\approx 115$ & $\begin{array}{l}\approx 121 \\
0.119\end{array}$ & $\approx 140$ & & \\
\hline$\infty$ (Meliga et al. 2009) & 116.9 & $\begin{array}{l}125.3 \\
0.121\end{array}$ & 143.7 & & \\
\hline$\infty$ (Chrust et al. 2010) & 116.92 & $\begin{array}{c}(124,125.2) \\
0.120\end{array}$ & $\begin{array}{c}{[142,143]} \\
0.118\end{array}$ & {$[165,170]$} & \\
\hline 50 (Present) & 120.5 & $\begin{array}{c}{[128.5,130]} \\
0.121\end{array}$ & $\begin{array}{c}{[140,141.5]} \\
0.114\end{array}$ & {$[185,190]$} & {$[210,215]$} \\
\hline 10 (Chrust et al. 2010) & 129.6 & $\begin{array}{c}(136.3,138.7) \\
0.115\end{array}$ & $\begin{array}{l}154.4 \\
0.114\end{array}$ & 188.8 & \\
\hline 10 (Shenoy \& Kleinstreuer 2008) & 135 & $\begin{array}{c}155 \\
0.113\end{array}$ & 172 & 280 & \\
\hline
\end{tabular}

TABLE 2. Critical Reynolds number and Strouhal number at different bifurcations. The results for thin disks are listed in the table so that $R e_{c 1}, R e_{c 2}$, and $R e_{c 3}$ are the onsets of the SS, RSB, and SW states respectively for all cases. Note that Chrust et al. (2010) and Shenoy \& Kleinstreuer (2008) did not define a threshold between the quasi-periodic state and chaotic state. Although the threshold between the quasi-periodic state and chaotic state is still difficult to find here, we propose that this bifurcation is at $R e_{c 5}$ based on our extensive numerical simulations. At $R e_{c 4}$, where the flow begins to lose its periodicity, our results shows good agreement with previous studies (Chrust et al. 2010). The parentheses denote the bistability interval and the square brackets correspond to the mean error margin.

symmetry is recovered. The $C_{z}-C_{y}$ diagram indicates that the lift force remained in the symmetry plane of the flow and is oscillating around a mean value of zero. The $C_{x}-C_{y}$ diagram shows a closed-path butterfly-like attractor, which implies that the periodicity of the flow and the frequency of the lift force is half of that of the drag force (similar to figure 5 in Auguste et al. 2010). As shown in figure 4(d), a secondary frequency occurs at $S t_{l}=0.034$, which is approximately one-third of the primary frequency $(S t=0.114)$ in the previous state (Chrust et al. 2010; Auguste et al. 2010). The $C_{x}-C_{y}$ diagram maintains the shape of a butterfly-like attractor, but no longer shows a single loop; this is typical of a quasi-periodic system (c.f. figure $8 a$ in Auguste et al. 2010). Finally, both the periodicity and the symmetry plane are lost when the flow became chaotic (figure $4 e$ ). This validation process provides a clear conclusion regarding the transition scenarios of a circular disk of $\chi=50$ placed normal to the incoming flow. These states and their most important characteristics agreed well with the results of previous studies. The critical Reynolds number and Strouhal number at different bifurcations are listed and compared in table 2. Our results are well located within the range between $\chi=\infty$ and $\chi=10$ published previously (Chrust et al. 2010). Based on the good agreement obtained, it is concluded that the present numerical approach is able to provide reliable results for the flow around an inclined disk. Validation of this method with experimental results (Calvert 1967) has also been previously reported (Tian et al. 2017a).

\section{Results}

In the present study, numerical simulations are conducted for 318 pairs of $(R e, \alpha)$, which are represented by the symbols shown in figure 5 . After careful examinations, five flow regimes in the considered parametric space are observed and denoted as: steady state 


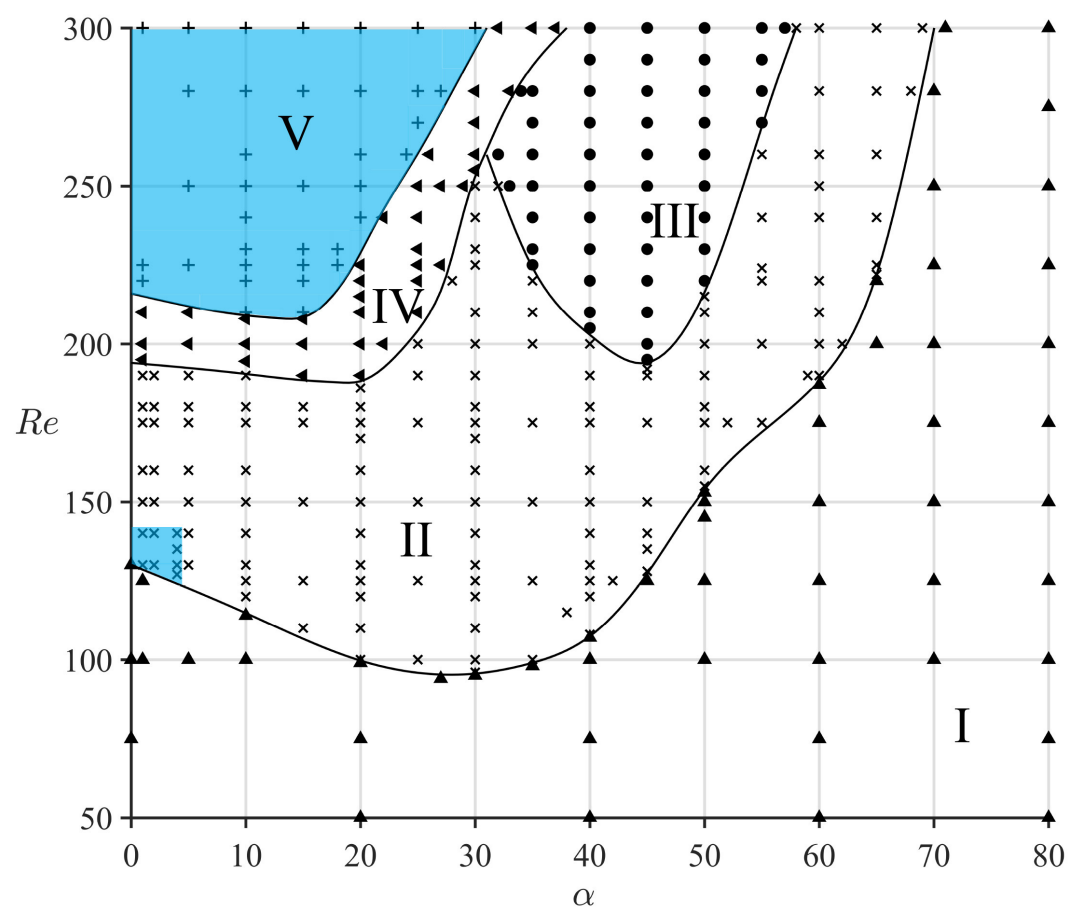

FIgURE 5. Classification of flow regimes behind a fixed inclined disk in the considered $R e-\alpha$ parametric space. I: steady state (SS), $\mathbf{\Delta}$; II: periodic state $(\mathrm{PS}), \times$; III, periodic state with a low frequency modulation (PSL), •; IV, quasi-periodic state (QP), ४; and V: chaotic state (CS), + . Except the blue regions, the flow always shows a fixed plane of symmetry selected by the inclination.

(SS), periodic state (PS), periodic state with a low frequency modulation (PSL), quasiperiodic state (QP), and chaotic state (CS). The thresholds between different regimes are identified by the solid lines. The Hopf threshold is obtained by the linear interpolation of force coefficients amplitude amplification/decay rates near it. The technical details could be found in Tian et al. (2017b). Although it is difficult to determine other boundaries accurately, careful examination is conducted to classify the cases by typical characteristics belonging to different regimes, e.g. non-coincident $C_{x}-C_{y}$ parametric plot in the QP area; loss of reflectional symmetry in the CS area; frequency peak band in the PSL area. Based on the extensive simulations, the error margin of these boundaries is less than 2 in Reynolds number at the same $\alpha$. Note that the bifurcations sequence at $\alpha=0^{\circ}$ is still observed when the disk is inclined. The inclinations tend to first promote the thresholds and then delay them. Any tiny inclination, changes the perfect axisymmetric flow configuration into a configuration of planar symmetry, resulting in a fixed reflectional symmetry plane of the wake, i.e. the plane of $z / D=0$ for all states, except two blue regions in figure 5 . One is located in the PS state area, which is well above the RSB state $\left(R e \approx 130-140, \alpha=0^{\circ}\right)$ but only appears when $\alpha$ is smaller than $4^{\circ}$. Here, a non-zero mean lift always emerges in the direction perpendicular to the plane of symmetry selected by the inclination. This phenomenon with respect to the weakly non-linear model has been reported and investigated in Chrust et al. (2015). Nevertheless, inclination recovers the reflectional symmetry of wake when $\alpha>4^{\circ}$. The other one is the whole area of chaotic regime, where the loss of reflectional symmetry is always conserved. In the following 


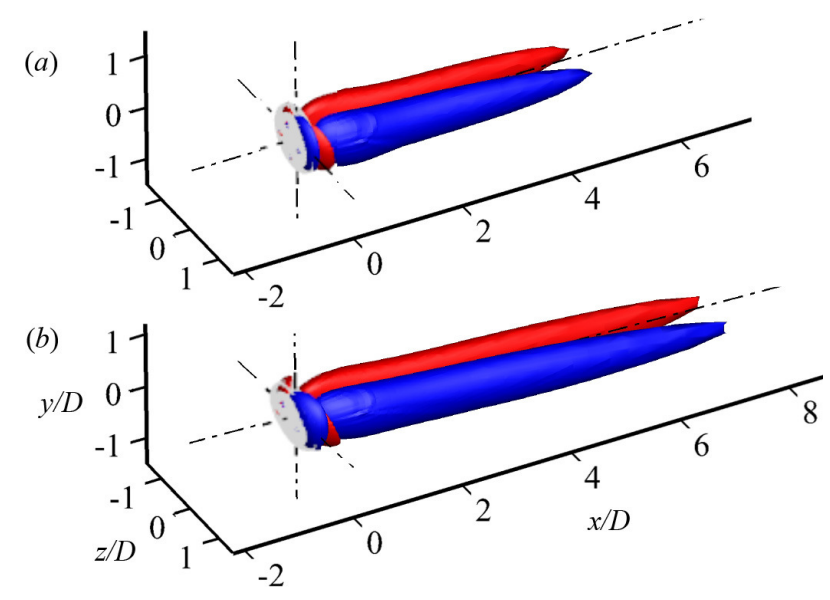

FIgURE 6. Sample vortical structure in the SS state. The disk is inclined by (a) $\alpha=1^{\circ}$; (b) $\alpha=10^{\circ}$ at $R e=100$. Iso-surfaces of the axial vorticity $\omega_{x}$ are of the same magnitude ( \pm $\left.0.1 U_{\infty} / D\right)$ and are represented by red and blue surfaces, respectively.

section, we presented representative cases for each flow regime to illustrate the wake transition behind a circular disk with varying $R e$ and $\alpha$ and discuss the questions raised in $\S 1$.

\subsection{Regime I: steady state (SS)}

As shown in table 2, when a thin circular disk of $\chi=50$ is placed normal to the incoming flow, a regular bifurcation at $R e_{c 1}=120.5$ is observed prior to the second Hopf bifurcation at $128.5<R e_{c 2}<130$; the wake changes from axisymmetry to planar symmetry before becoming unsteady. As shown in figure 6, a small inclination of $\alpha=1^{\circ}$ already results in a new symmetry plane of $z / D=0$ at $R e=100$. Therefore, for the inclined cases around the primary bifurcation, irrespective of the steady axisymmetric flow or steady planar symmetric flow, any inclination results in a double-threaded and counter-rotating axial vorticity (similar to figure 4 in Shenoy \& Kleinstreuer 2008). A steady and positive lift force in the $y$-axis direction appears for all cases in the SS state. The inclination as a perturbation introduced to the perfectly axisymmetric system yields a planar symmetry flow pattern, which is found to be the exclusive flow pattern below the boundary of Hopf instability. The primary bifurcation disappears. It is worth mentioning that the imperfect configuration could strengthen the intensity of the streamwise vortices (see figure 6), which is also reported by Chrust et al. (2015).

\subsection{Regime II: periodic state $(P S)$}

The first bifurcation of inclined disks is of Hopf type. It is numerically investigated here up to $R e=300$. As shown in table 3 , the $R e_{c}$ and $S t_{p}$ show good agreement with those previously published (Chrust et al. 2015) at $\alpha<35^{\circ}$, even though their disk is infinitely thin. It is reasonable to believe that at small $\alpha$ the appearance of disk edge and thickness do not introduce significant effects, e.g. acting as strong source of vorticity like the tips of foils and wings or influencing critical Reynolds number, at least for thin bodies. As shown in figures 7 and 8, four specific cases at $R e=175$ in the PS state area are chosen as the samples. From figures $7(a-d)$ and $8(a-d)$, the three-dimensional (3D) vortical structure sketches and $C_{z}-C_{y}$ diagrams verify the aforementioned conclusion that the reflectional symmetry of the wake with respect to the plane of $z / D=0$ is always 


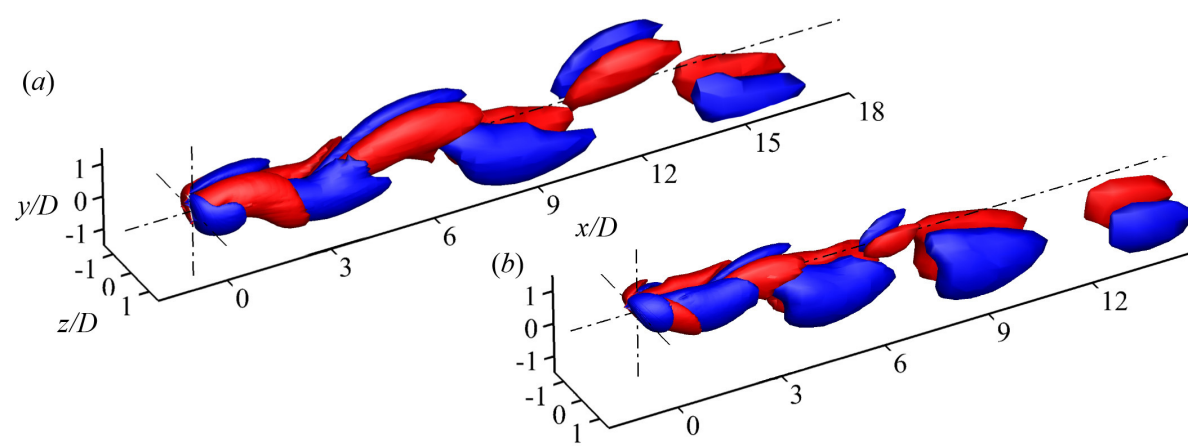

(c)

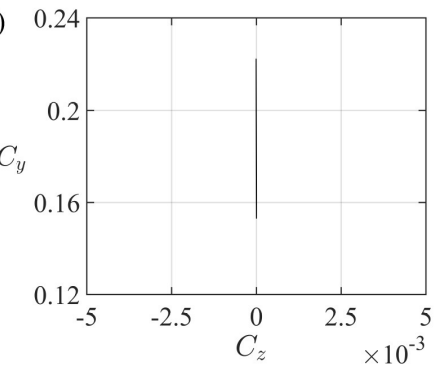

(e)

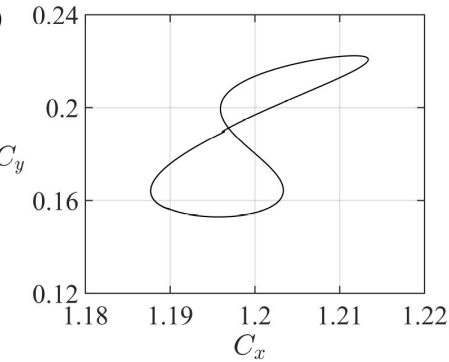

$(g)$

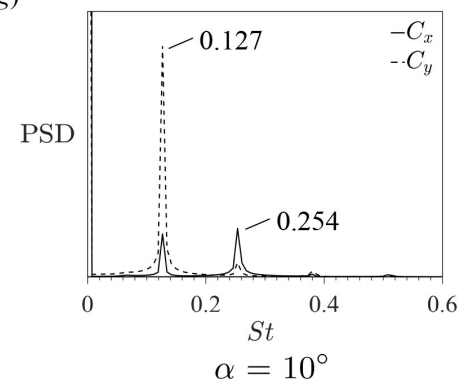

(d)

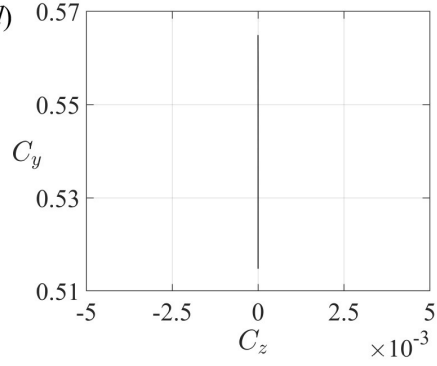

(f)

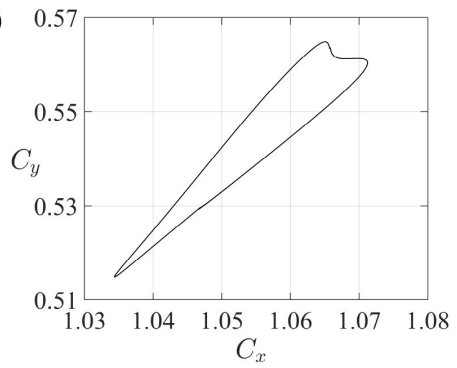

$(h)$

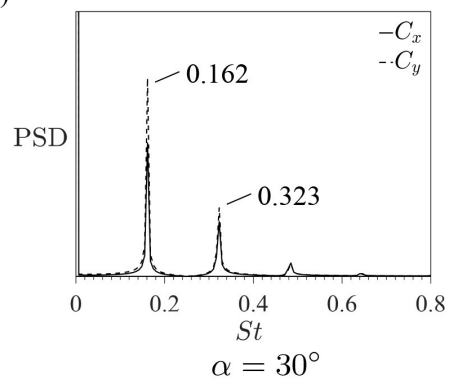

Figure 7. Part I: sample characterisations of PS state. The disk is inclined by $\alpha=10^{\circ}$ (left column); $\alpha=30^{\circ}$ (right column) at $R e=175$. (cf. Part II in figure 8). (a-b) $\omega_{x}$ iso-surfaces are shown at the level of $\pm 0.1 U_{\infty} / D$. $(c-d) C_{z}-C_{y}$ diagrams indicate the reflectional symmetry of the wake. $(e-f) C_{x}-C_{y}$ diagrams imply the periodicity of the force coefficients. $(g-h)$ Power spectra density (PSD) of $C_{x}$ and $C_{y}$. One dominating frequency, corresponding to the vortex shedding frequency, and its higher harmonics are observed, further indicating the periodicity of the force coefficients. 


\begin{tabular}{cccccc} 
& \multicolumn{2}{c}{ Chrust et } & al. $(2015)$ & & \multicolumn{2}{l}{ Present study } \\
\cline { 2 - 3 }$\alpha$ (deg.) & $R e_{c}$ & $S t_{p}$ & & $R e_{c}$ & $S t_{p}$ \\
0 & 125.2 & 0.120 & & 130.0 & 0.121 \\
10 & 114.3 & 0.111 & & 114.8 & 0.110 \\
20 & 96.4 & 0.124 & & 99.7 & 0.123 \\
30 & 95.3 & 0.148 & & 95.6 & 0.145 \\
40 & 104.9 & 0.183 & & 107.3 & 0.177 \\
50 & 132.6 & 0.226 & & 154.2 & 0.223 \\
60 & - & - & & 188.5 & 0.261
\end{tabular}

TABLE 3. Comparison of the critical Reynolds number $R e_{c}$ and Strouhal number corresponding to the frequency of primary vortex shedding $S t_{p}$ on the Hopf threshold. $R e_{c}$ and $S t_{p}$ are all normalized by the short axis of projection in the streamwise direction $D \cos \alpha$ to compare conveniently.

conserved in this regime. Moreover, periodic vortex shedding is well demonstrated. With increasing $\alpha$, the wake flow of the inclined disk is oriented towards the trailing side rather than the streamwise direction and the wavelengths decrease. Considering the $C_{x^{-}}$ $C_{y}$ diagrams shown in figures $7(e-f)$ and $8(e-f)$, although the loops have different shapes, a single closed loop is formed, indicating the periodicity of the flow. With increasing $\alpha$, the previous primary frequency of $C_{x}$ (solid black line) gradually gives way to a new dominant frequency until these two force coefficients shares the same dominant frequency; this process could be seen in figures $7(g-h)$. Then, the dominant frequency is always identical for the drag and lift force (see figures $8 g$ - $h$ ). If $\alpha$ keeps growing, the magnitude of the higher harmonics recesses gradually until the flow becomes perfectly harmonic near the boundary of the SS state, as shown in figure $8 h$.

The streamlines behind the disk could further explain these phenomena from the prospective of vortex shedding patterns. Behind an inclined circular disk, there are two toroidal vortices generating from the leading and trailing edges, respectively. As shown in figure 9 , the two toroidal vortices alternatively grow or shrink to feed each other and the downstream wake, resulting in a periodic flow. To be specific, as the lower vortex grows and sheds (figures $9 a-d$ ), the upper one shrinks to feed it until approaching a local shortest length at figure $9(d)$. Then the upper one grows at rapidly increasing speed until a local largest length at figure $9(f)$ (see movie 1 in the supplementary animations). This process is similar to the wake behind a circular disk of $\chi=5$ normal to the flow at $R e=300$ (Yang et al. 2015). Instead of developing arbitrarily at $\alpha=0^{\circ}$, the flow behind inclined disk is regulated and the periodic vortex shedding is promoted so that at the same Reynolds number the wake is more likely to become periodic. Consequently, the threshold corresponding to steady state - periodic state transition (the section of $\alpha<30^{\circ}$ ) is advanced. When $\alpha$ increases, the upper toroidal vortex almost stops growing and shrinking, instead, it remains stably to the disk surface, leaving only the lower one shedding periodically (see movie 2 in the supplementary animations). Similar phenomenon had been reported in aerodynamics that the leading edge vortices are found to be stably attached and provide lift force for fixed, flapping or hovering wings with some angles of attack (Dong et al. 2006; Taira \& Colonius 2009). It is found that the larger $R e$ is, the larger $\alpha$ is required to make upper toroidal vortex remain stable. As a result, the threshold of Hopf bifurcation corresponding to periodic state - steady state transition $\left(\alpha>30^{\circ}\right)$ is also delayed.

Strouhal number obtained from extensive cases is illustrated here. As shown in 


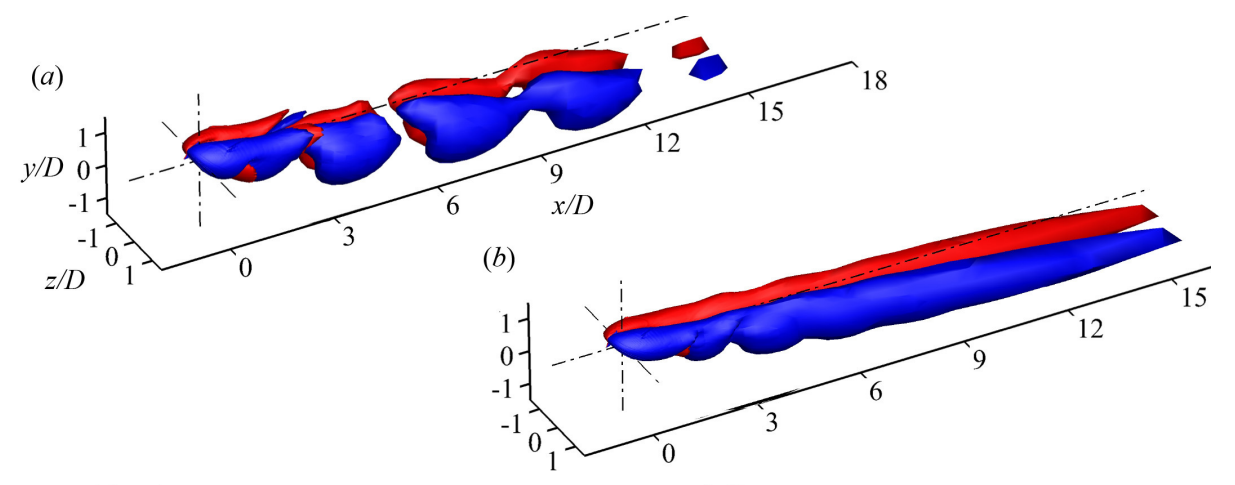

(c)
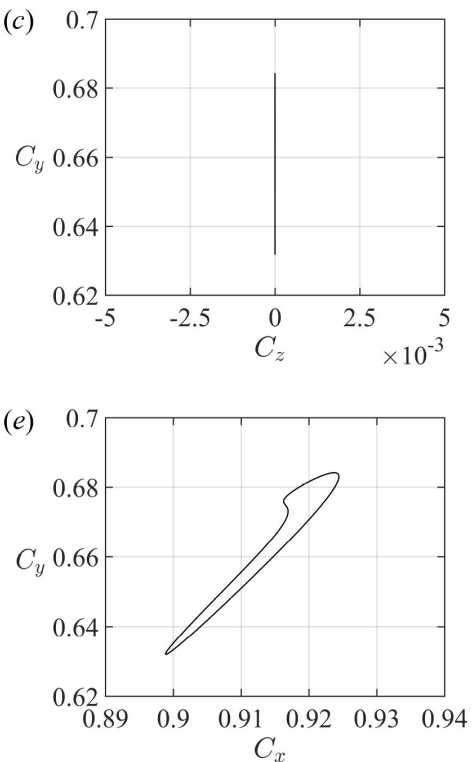

$(g)$

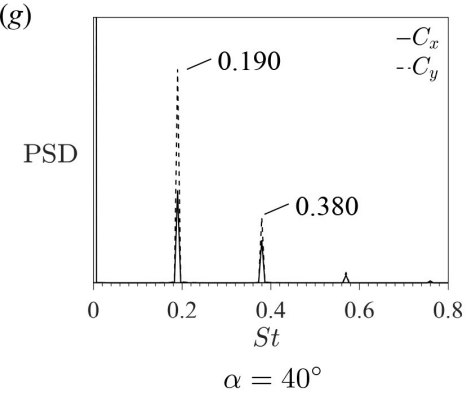

$(d)$

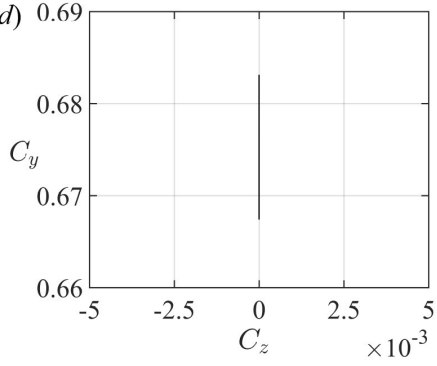

(f)

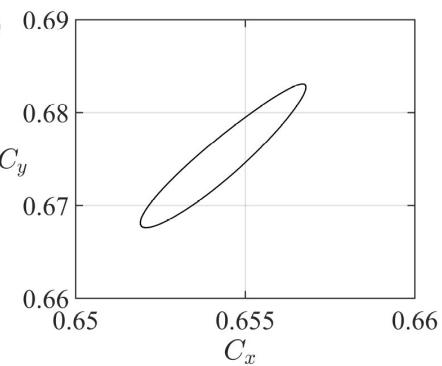

(h)

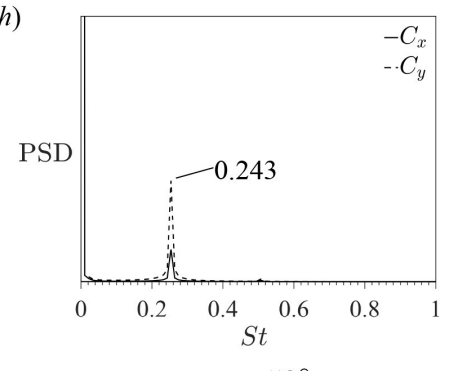

FiguRE 8. Part II: sample characterisations of PS state. The disk is inclined by $\alpha=40^{\circ}$ (left); $\alpha=52^{\circ}$ (right) at $R e=175$. It can be seen that the reflectional symmetry and periodicity of the force coefficients are conserved over the whole regime. The Strouhal number $S t_{p}$ goes up with the increase of $\alpha$, while the amplitudes of higher harmonics recess.

figure 10, our results are in very good agreement with previous DNS data for $\alpha=10^{\circ}$, $20^{\circ}$, and $30^{\circ}$. When $\alpha$ is constant, the $S t_{p}$ increases slightly with increasing $R e$. And the Strouhal number is independent of $R e$ in the chaotic regime (corresponding to the cases of $R e>240$ when the disk is inclined by $\alpha=10^{\circ}, 20^{\circ}$, and $30^{\circ}$ ) (Chrust et al. 2015). However, for a given Reynolds number, $S t_{p}$ increases significantly with increasing $\alpha$. 

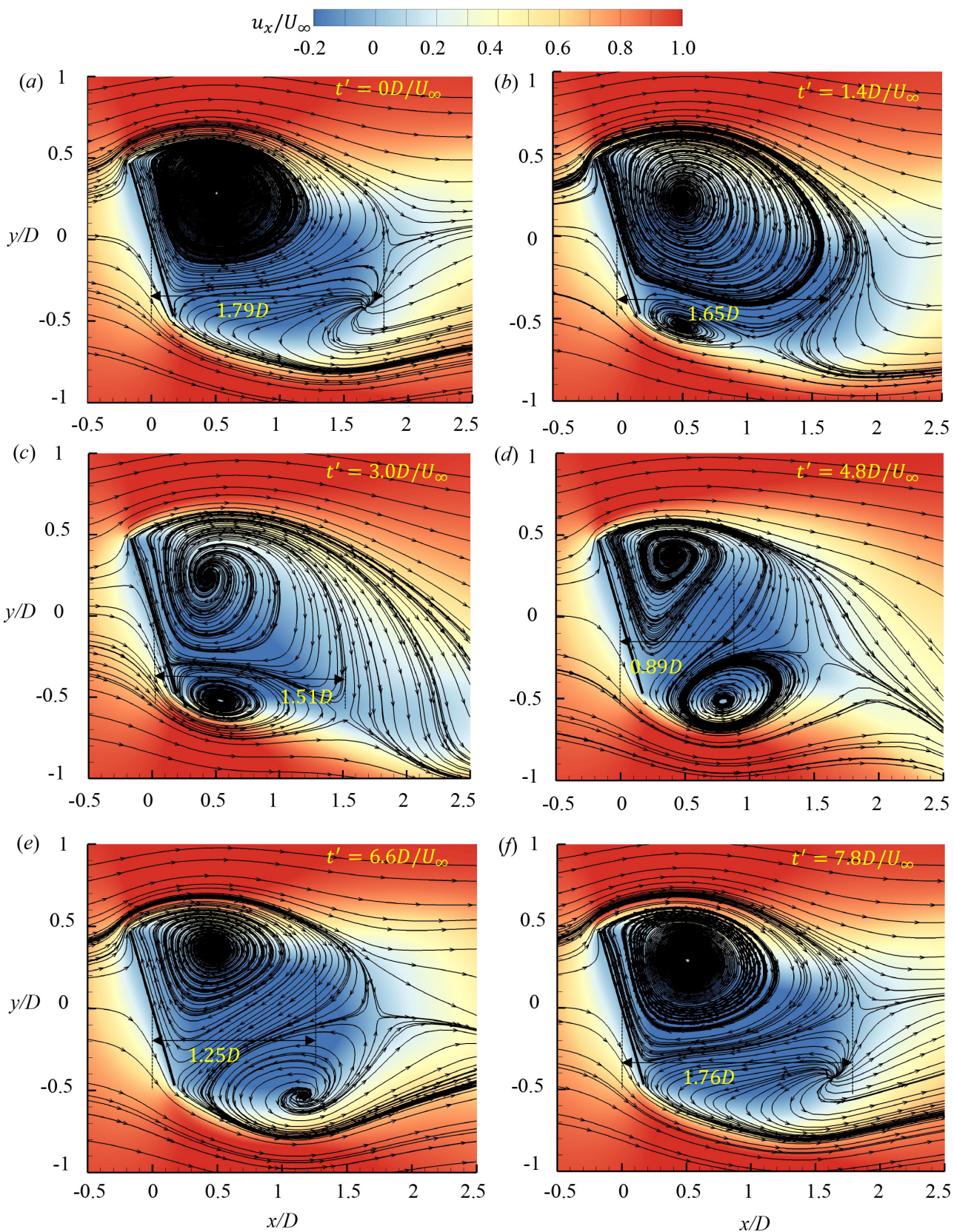

FiguRE 9. Instantaneous streamlines in the plane of $z / D=0$ at $R e=150$ when the disk is inclined by $\alpha=20^{\circ}$ (PS State). Six sketches happen successively throughout a whole period equal to $1 / f D / U_{\infty}$. The diameter of the upper toroidal vortex which is defined by the distance from the disk centre to the end of upper recirculation region, has been labeled in each plot.

\subsection{Regime III: Periodic state with a low frequency modulation (PSL)}

In the considered $R e-\alpha$ space, a unique flow regime with intermediate $\alpha$ range (about $30^{\circ}-55^{\circ}$ ) is observed when $R e$ is larger than 195 . Besides the periodic vortex shedding, another pronounced low frequency is also observed. Similar flow pattern is reported for 


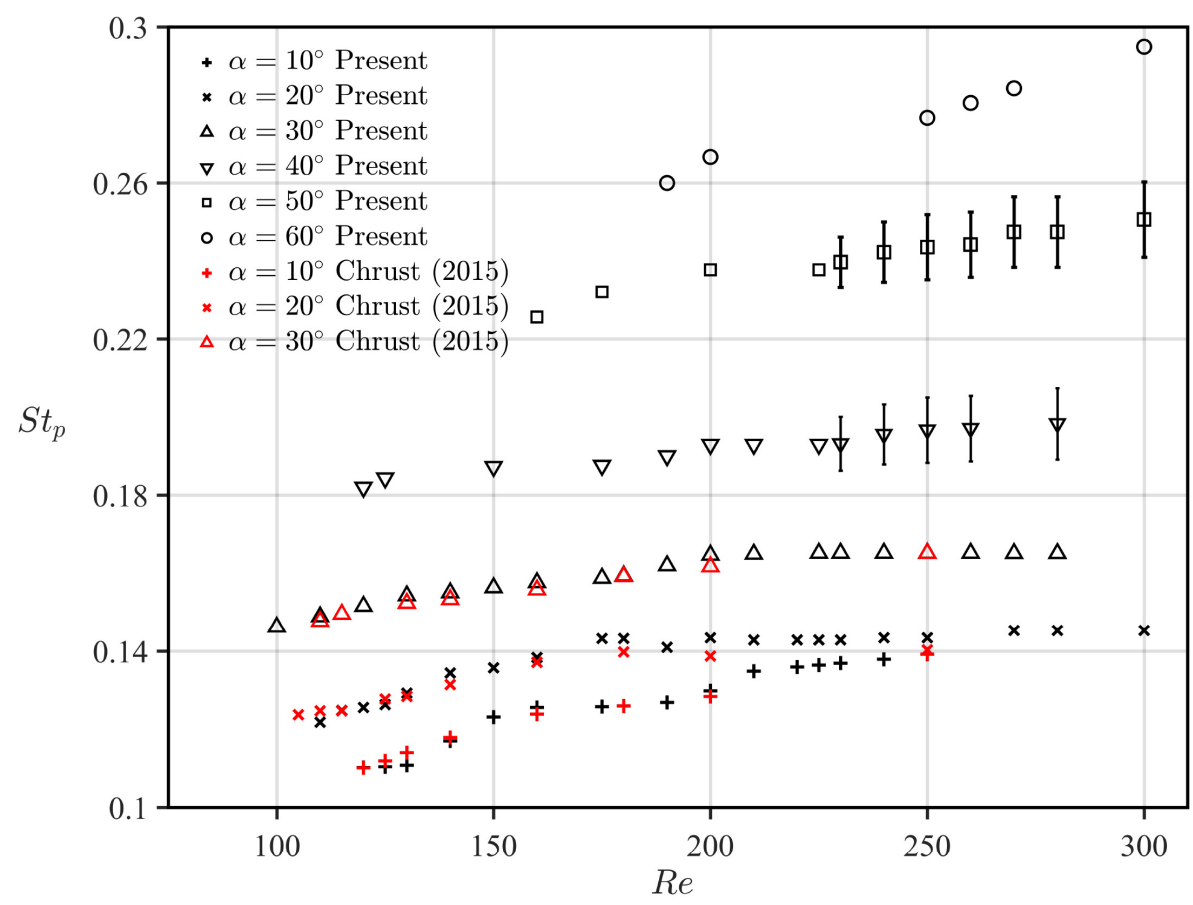

FIgURE 10. Strouhal number $S t_{p}$ as a function of Reynolds number for different inclination angles $\alpha$. The results of our study (black markers) are compared to those from a previous one (Chrust et al. 2015) (red markers). St $t_{p}$ is normalized by $D \cos \alpha$ for comparison. When the disk is inclined by $\alpha=40^{\circ} ; \alpha=50^{\circ}$ at $R e \geqslant 230$, the cases locate in the PSL state area, where the dominant frequency shows a wide band rather than a single peak. Therefore, $S t_{p}$ are represented by error bars to delimit the errors.

flow past a disk inclined by 45 and $50^{\circ}$ at $R e=500$ (Tian et al. $2017 a$ ). This state inherits key features of the PS state, while the flow is modulated by a low frequency component; therefore, it is named 'periodic state with a low frequency modulation' (PSL). Several main features of the PSL state are shown in figure 11. From the $3 \mathrm{D}$ vortical structure and $C_{z}-C_{y}$ diagram, the flow shows reflectional symmetry and a double-threaded vortex with periodic shedding is observed, generating only from the trailing edge. Figure 11(b) clearly presents this low frequency modulation, whose period is significantly longer than that of the primary vortex shedding. As shown in figure 12, the primary frequency modulated by a low frequency is again clearly observed in the pressure and velocity fields at various downstream locations.

Recalling the validation work that when the flow behind disk begins to lose the plane of symmetry and enters the quasi-periodic regime at $R e \approx 190$, a low frequency at $S t=0.034$ is observed. Previous studies attributed this to the irregular rotation of the azimuthal location of large-scale vortex shedding (Tomboulides \& Orszag 2000; Shenoy \& Kleinstreuer 2008) and the low frequency pumping motion of the recirculation region (Berger et al. 1990). It is further reported that these two physical origins resulted in different low frequencies at $S t=0.02$ and $S t=0.03(\chi=5, R e=300)$ (Yang et al. 2015). Here, the low frequency at $S t_{l} \approx 0.026$ is obtained in the PSL state (figure 11e); the primary vortex shedding frequency and its higher harmonics are also clear, appearing as a wide frequency band rather than a single well-defined peak. It is noted that the low 
(a)

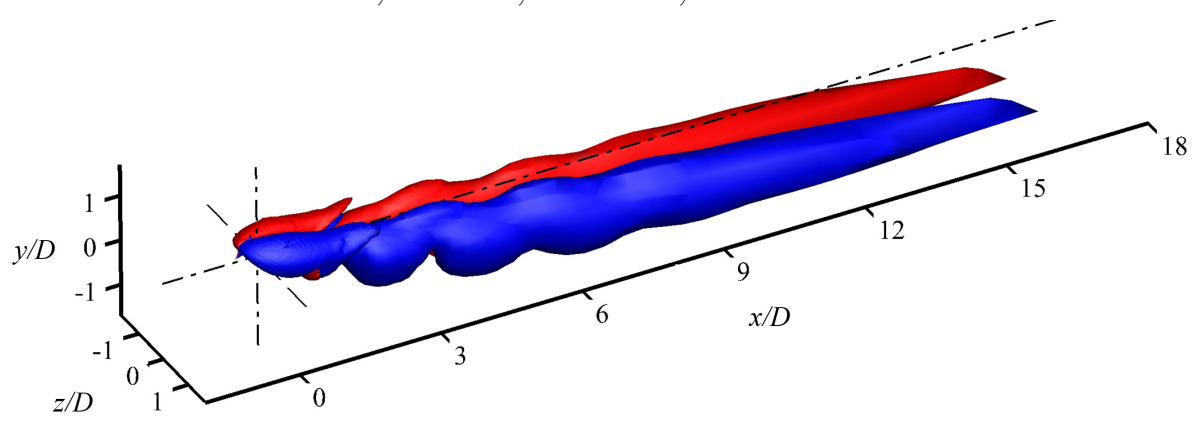

(b)

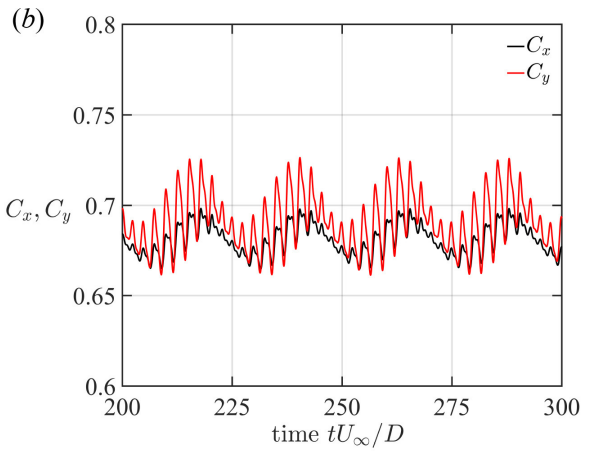

(d)

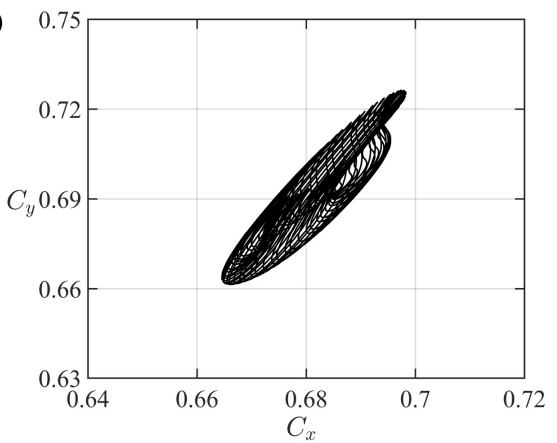

(c)

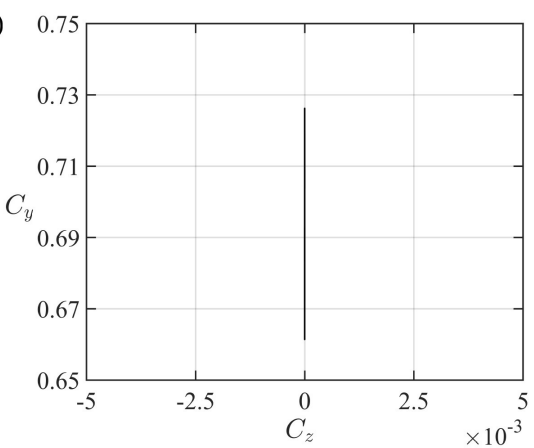

$(e)$

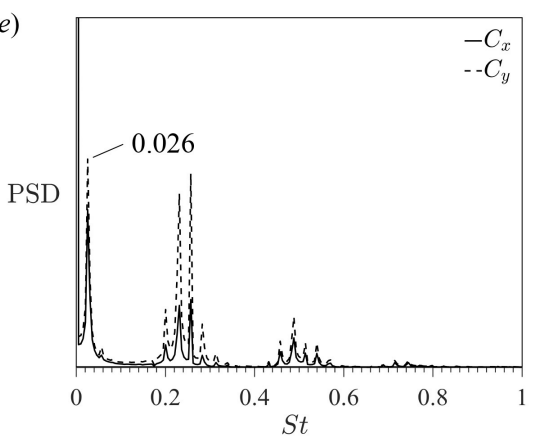

Figure 11. Sample characterisations of the PSL state. The disk is inclined by $\alpha=50^{\circ}$ at $R e=250$. (a) $\omega_{x}$ iso-surfaces are all demonstrated for $\pm 0.1 U_{\infty} / D$. Reflectional symmetry is observed. (b) Time traces of the force coefficients, $C_{x}$ and $C_{y}$. It is observed that periodic vortex shedding is associated with a low frequency modulation. (c) $C_{z}-C_{y}$ diagram indicated reflectional symmetry of the wake. $(d) C_{x}-C_{y}$ diagram shows a complicated pattern, indicating complex frequency components of the force coefficients. (e) PSD of $C_{x}$ and $C_{y}$. The low frequency modulation and frequency bands (with harmonics) are all obtained.

frequency is much more pronounced in PSL state than QP state (c.f. figure 4d). Moreover, $S t_{l}$ is only about one-tenth to the $S t_{p}$ while this ratio is about one-fourth in QP state. Both periodic vortex shedding and a similar pumping motion over a much longer time scale are observed in the $3 \mathrm{D}$ visualization of the vortical structure (see movie 3 in the supplementary animations). However, the pumping motion in PSL state shows some differences. As shown in figure 13, the vortex on the trailing edge continues to shed and gives rise to the main oscillation. Nevertheless, the toroidal vortex on the leading edge neither grows and shrinks periodically with the trailing edge one nor stably attaches to 

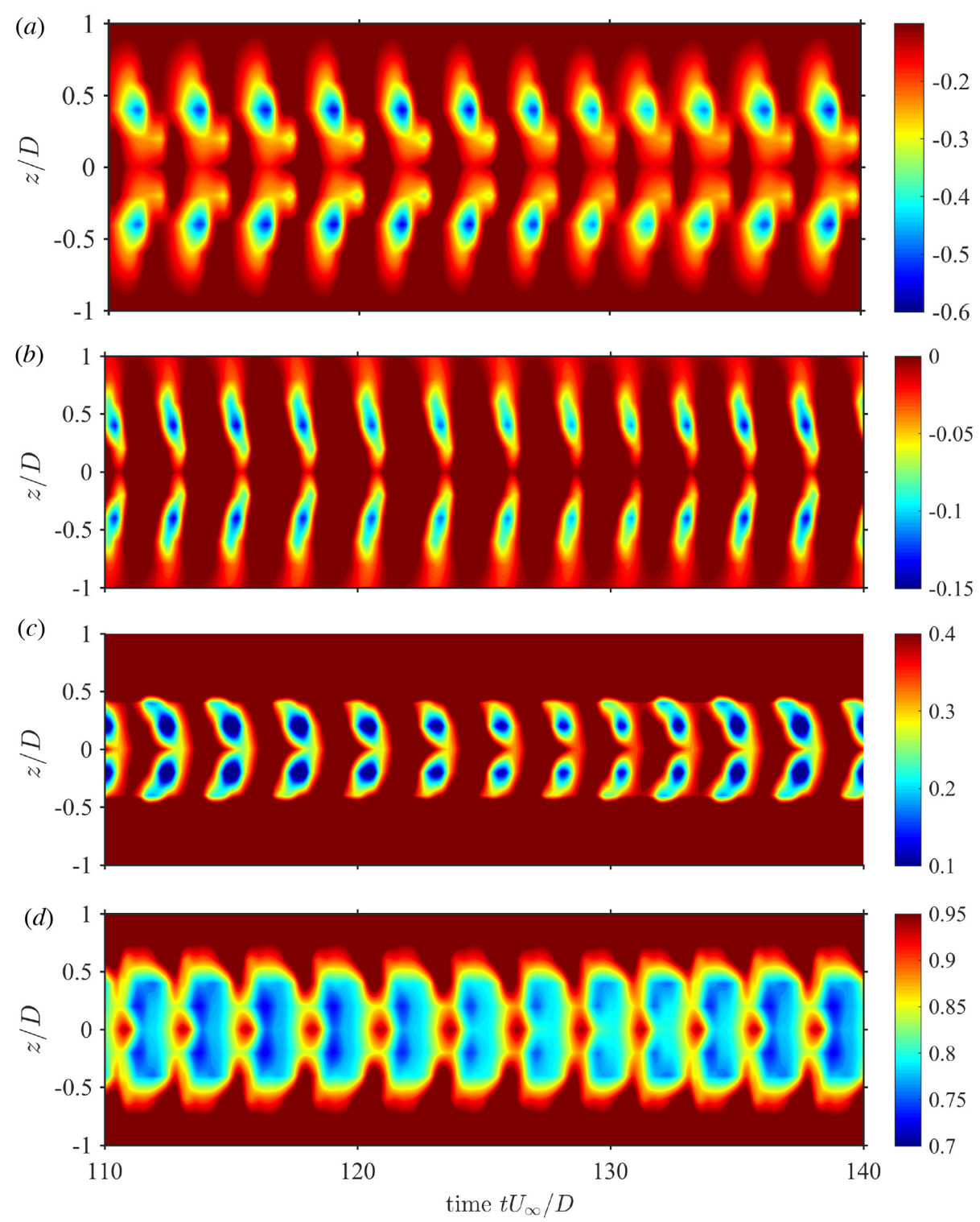

Figure 12. Time dependency of $(a-b)$ pressure coefficient $C_{p} ;(c-d)$ dimensionless axial velocity $u_{x} / U_{\infty}$. The disk is inclined by $\alpha=50^{\circ}$ at $R e=250$. One should note that $(a, c)$ demonstrate the line of $x / D=1, y / D=0$, c.f. $(b, d)$ the line of $x / D=2, y / D=0$. The pressure coefficient $C_{p}$ is defined as $C_{p}=\left(p-p_{\infty}\right) /\left(0.5 \rho U_{\infty}^{2}\right)$, where $p_{\infty}$ is the reference pressure at the centre of the inlet boundary. low frequency oscillations are observed in the sketches at different downstream locations.

the disk. Instead, it presents a similar low frequency pumping motion alone. Specifically, in figures 13(a-c), when the lower toroidal vortex keeps growing, the upper one also grows. This process is in contrast to the process in PS state (c.f. figure 9), implying that the pumping motion of the upper toroidal vortex is independent of the primary vortex shedding. It is most likely the intermediate $\alpha$ range of PSL state gives rise to an intermediate motion status of leading edge vortex. This type of motion is different from the previous reported pumping motion, which is provided by leading and trailing 

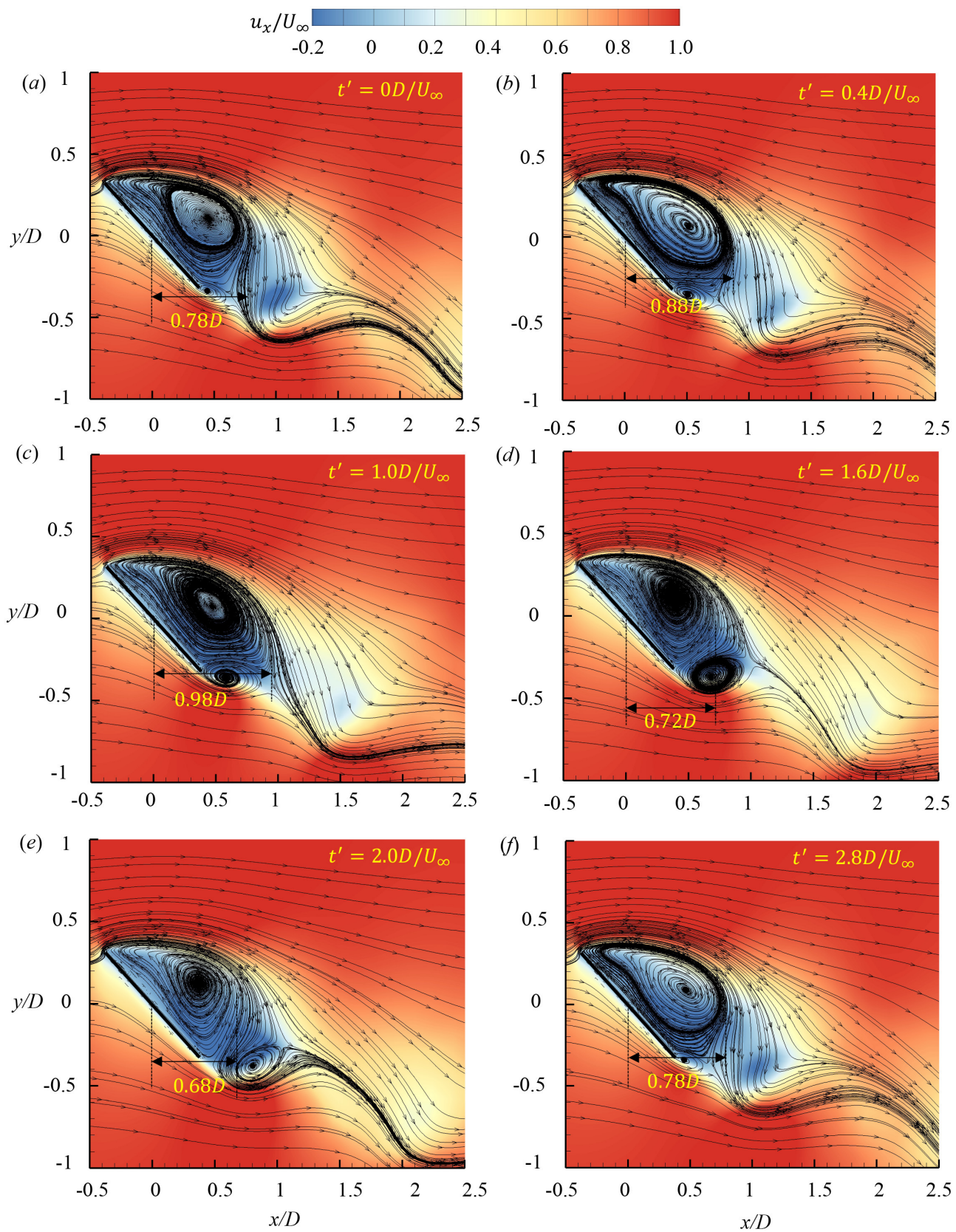

FIGURE 13. Instantaneous streamlines in the plane of $z / D=0$ at six successive time instants. The disk is inclined by $\alpha=50^{\circ}$ at $R e=250$ (PSL state). Note that the upper toroidal vortex oscillates independently of the periodic shedding, i.e. neither grows and shrinks with lower one (c.f. figure 9) nor remains stably.

edges together. It is reasonable to believe such difference leads to the aforementioned discrepancy in the amplitude of low frequency and the ratio value of $S t_{l} / S t_{p}$. 


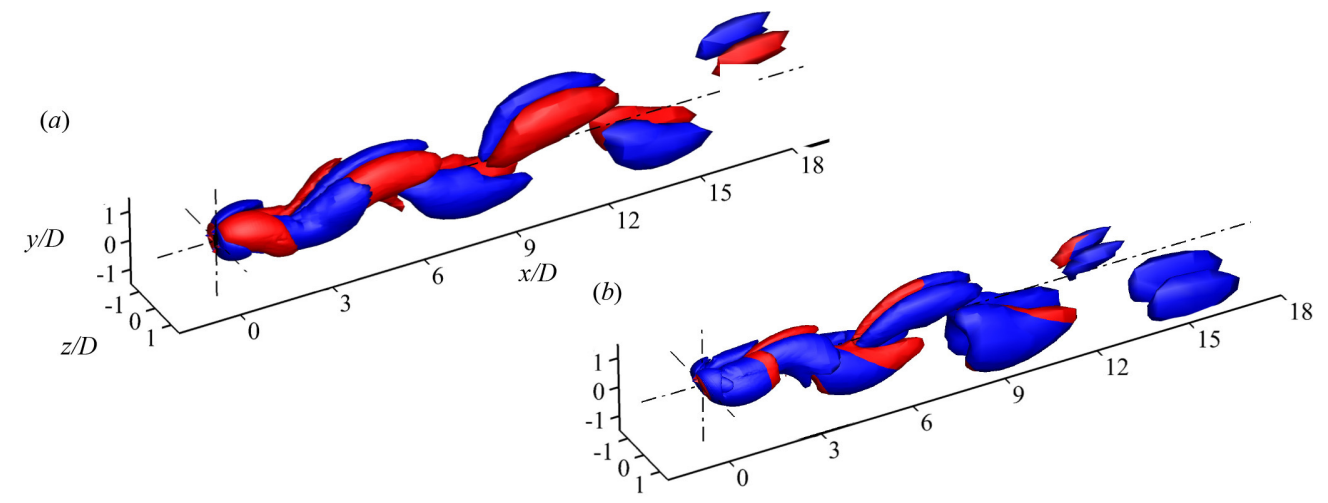

(c)

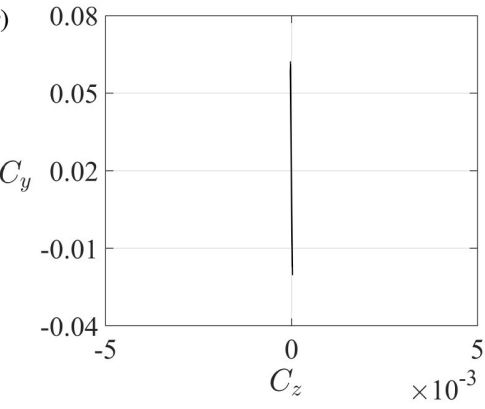

(e)

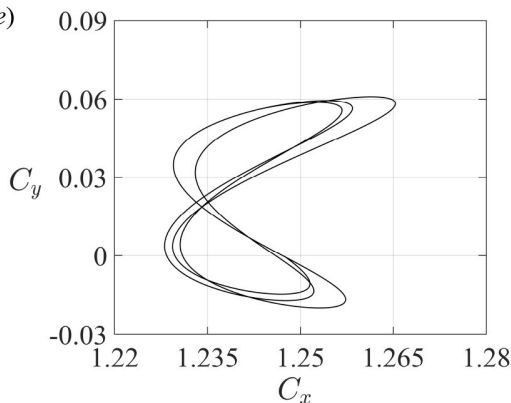

$(g)$

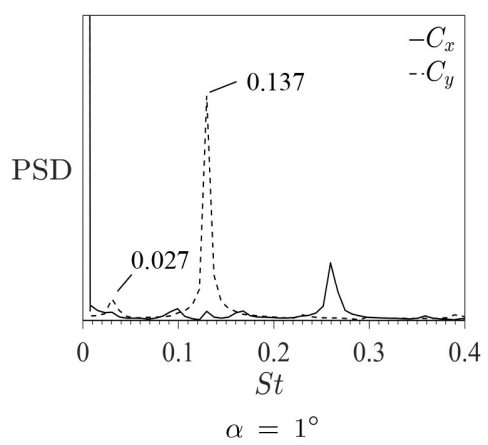

(d)

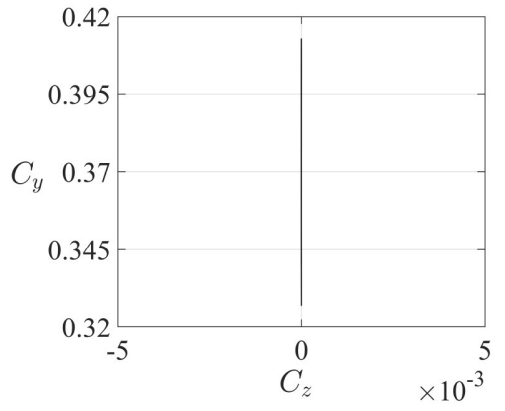

$(f)$

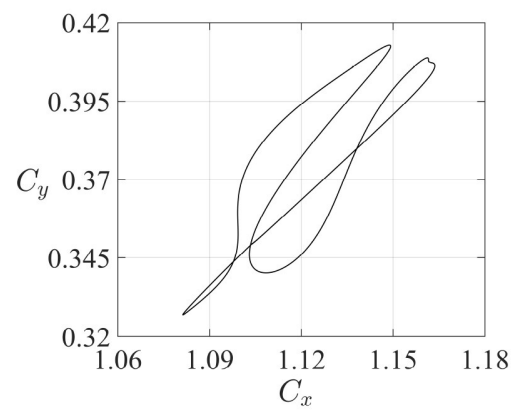

(h)

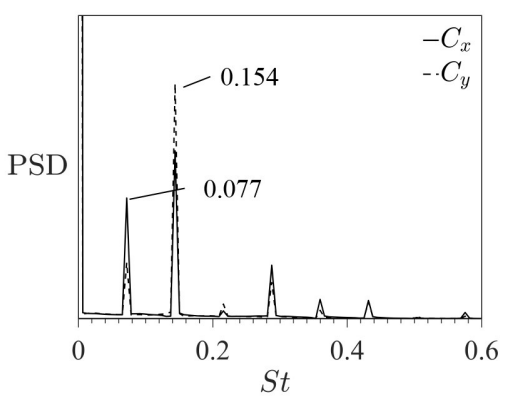

Figure 14. Sample characterizations of QP state. The disk is inclined by $\alpha=1^{\circ}$ (left); $\alpha=20^{\circ}$ (right) at $R e=210$. When $\alpha=0^{\circ}$, the plane of symmetry is arbitrary (see figure $4 d$ ). At $\alpha=1^{\circ}$, the reflectional symmetry of the wake is immediately recovered and conserved. Quasi-periodic features are observed in the $C_{x^{-}} C_{y}$ diagram and PSD. 


\subsection{Regime IV: Quasi-periodic state $(Q P)$}

Before flow behind a perpendicularly placed disk develops into a chaotic state, a state of quasi-periodicity pioneers. When the disk is inclined, this QP state is conserved for small inclination area ( $\alpha$ is approximately less than $30^{\circ}$ ). As shown in figure $4(c)$, the plane of symmetry is arbitrary, but stable at $R e=210$ when the disk is placed normal to the flow. Once it is inclined by $\alpha=1^{\circ}$, the reflectional symmetry of the wake with respect to the plane of $z / D=0$ is immediately recovered and conserved in the whole regime (see figures $14 c-d$ ). This is the same behaviour as that happening at the primary bifurcation. A low frequency component of $S t_{l} \approx 0.027$ is also observed when the disk is inclined by $\alpha=1^{\circ}$, and the ratio of $S t_{l}$ to $S t_{p}$ is also around $1 / 4$, implying the same physics (see figure $14 \mathrm{~g}$ ). When $\alpha$ goes up, this ratio develops towards a 1:2 'lock-in' state with primary frequency as shown in figure $14(h)$. Almost perfect ratio and phaselocking resulted in the single loop observed in the $C_{x^{-}} C_{y}$ diagram (see figure $14 f$ ). A similar phenomenon is previously reported (see figure $8 b$ in Auguste et al. 2010) where the flow returned to a periodic state at $R e=230$ after chaotic behaviour is observed at $R e=220$. After examination of the $3 \mathrm{D}$ plot of the vortical structures of the QP state (see movie 4 in the supplementary animations), it is reasonable to believe that this low frequency is also attributed to the pumping motion of the leading and trailing edges vortices together, the same physics of at $\alpha=0^{\circ}$ and $1^{\circ}$. Inclination selects plane of symmetry for the wake, regulates the flow and induces the periodic vortex shedding behind the disk, consequently the Hopf threshold is advanced in PS state. Similarly, in QP state the pumping of recirculation region is also stimulated, intensified and forced to move in resonance with the periodic vortex shedding. However, this exact 1:2 'lock-in' does not last long and the low frequency amplitude significantly decreases when $\alpha$ keeps increasing to the threshold of the PS state. Over this boundary, only the primary vortex shedding frequency and its harmonics leave, implying a periodic flow.

\subsection{Regime V: Chaotic state (CS)}

For a circular disk placed perpendicularly to the incoming flow, both the periodicity and reflectional symmetry of the wake disappear at the threshold of chaos, which is at $R e_{c 5}=$ $[210,215]$ here (see table 2). As shown in figure 15, the flow is always chaotic irrespective of the inclination angles, meaning that the bifurcation always leads a dominating position to control the flow in the CS state area; hence, no magnitude of inclination can recover the reflectional symmetry. As shown in figures 16(a,c,e), force coefficients all present irregular traces as well. Nevertheless, when $\alpha$ grows up to $30^{\circ}, C_{z}$ explores a much narrower range, implying that inclination still has some effect on the flow in this state. The vortices evolve chaotically along both $y$-axis and $z$-axis when disk is inclined by $\alpha=0^{\circ}$ and $1^{\circ}$, and the amplitudes of $C_{y}$ and $C_{z}$ are comparable in figures $16(a, c)$, showing a combined effect. Even though vortices are still irregular when disk is inclined by $\alpha=30^{\circ}$, their attachment orientation remains fixed. The irregular oscillation of $C_{z}$ is much more significant than that of $C_{y}$, which is attributed to the large inclination angle. This phenomenon can be further confirmed by the irregular azimuthal motion of the vortex shedding. As shown in figure $16(b)$, continuous rotation of the vortex shedding is observed when $\alpha=0^{\circ}$ (similar to figure $11 b$ in Shenoy \& Kleinstreuer 2008). The rotation arbitrarily changes direction and shows complete rotation around the circumference. When $\alpha=1^{\circ}$, although the vortex shedding is still rotating, it is limited to a narrow range, and the rotation oscillates around a plane of symmetry (see figure $16 \mathrm{~d}$ ). When disk is inclined by $\alpha=30^{\circ}$ (figure $16 f$ ), the flow is restrained to rotate around the plane of $z / D=0$ within $\pm 3^{\circ}$, which is apparently determined by the inclination. We conclude that, in the turbulent 
(a)

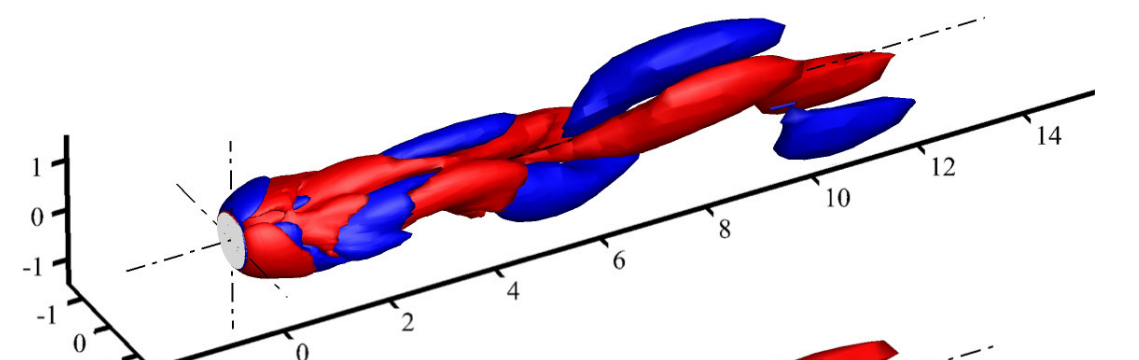

(b)

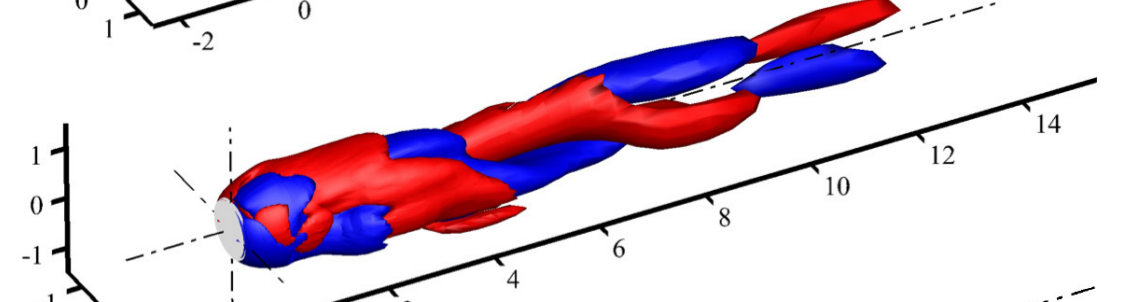

(c)

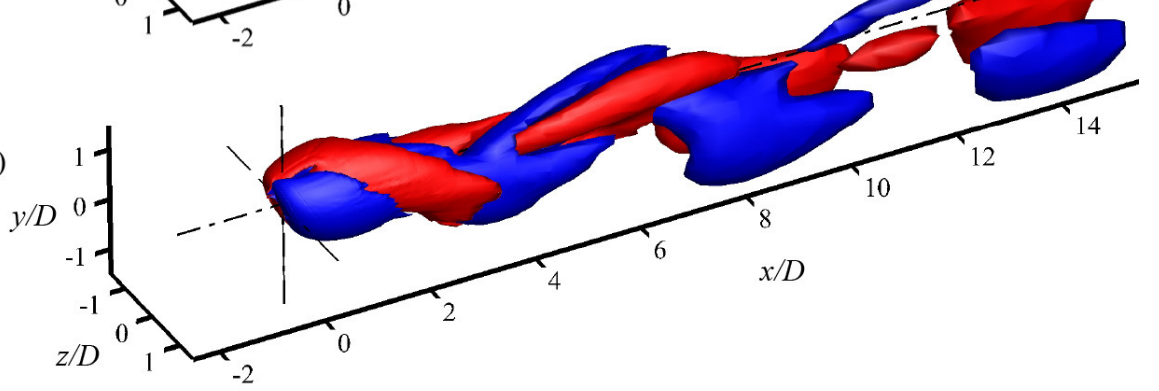

FIgURE 15. Vortical structures identified using $\omega_{x}= \pm 0.1 U_{\infty} / D$. The diks is inclined by $(a)$ $\alpha=0^{\circ} ;(b) \alpha=1^{\circ} ;(c) \alpha=30^{\circ}$ at $R e=300$. Irregular wake is observed for all three cases. Nevertheless, with increasing $\alpha$, the flow is regulated and reflectional symmetry with respect to the $z / D=0$ plane emerged gradually.

regime, the inclination has some control over the wake as well, although the bifurcation is always dominant.

\subsection{Wake instability of the fixed inclined disk and the freely falling disk}

Zhong \& Lee (2012) experimentally investigated the wake behind a thin disk $(\chi>40)$ falling vertically in quiescent water at low Reynolds numbers. In their study, the disk is falling in a rectilinear path with no significant rotation of the body (corresponding to the SV state), i.e. $I^{*}$ is not important in describing the motion of the disk and $R e$ is the only control parameter. Under such circumstances, the wake also experience a series of bifurcating transitions, and is similar to the evolution of the wake behind a fixed disk: the transition from an axisymmetric wake to the SS state at the first regular bifurcation $\left(R e_{c 1} \approx 105\right)$ and then the Hopf bifurcation occurred at $R e_{c 2} \approx 118$, leading to the RSB mode. Although these critical Reynolds numbers are both slightly smaller than those obtained in the present study, the transition and vortex shedding patterns indicated the similarity between them. Furthermore, when the disk is inclined, the bifurcations will be advanced so that the discrepancy further decreased, indicating more reasonable connections between a barely rotating freely falling disk and a fixed inclined disk. However, it is not to say that the mechanism of the freely falling disk can be considered as that of a fixed inclined disk. 
(a)

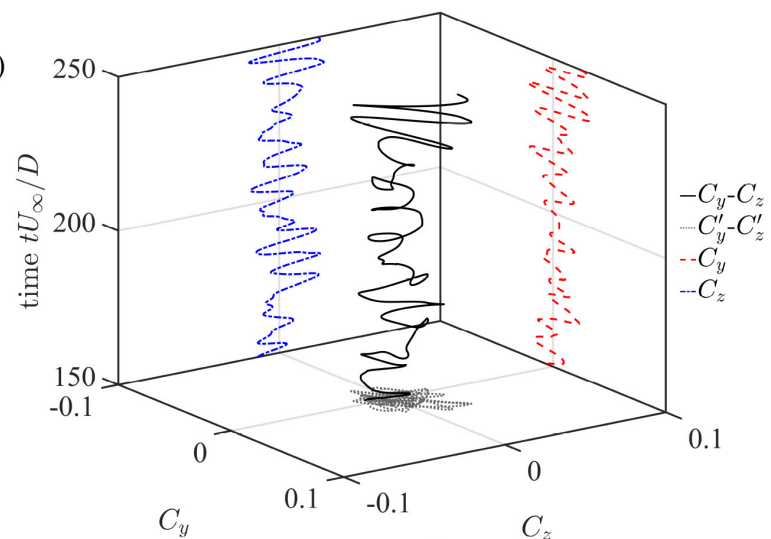

(c)

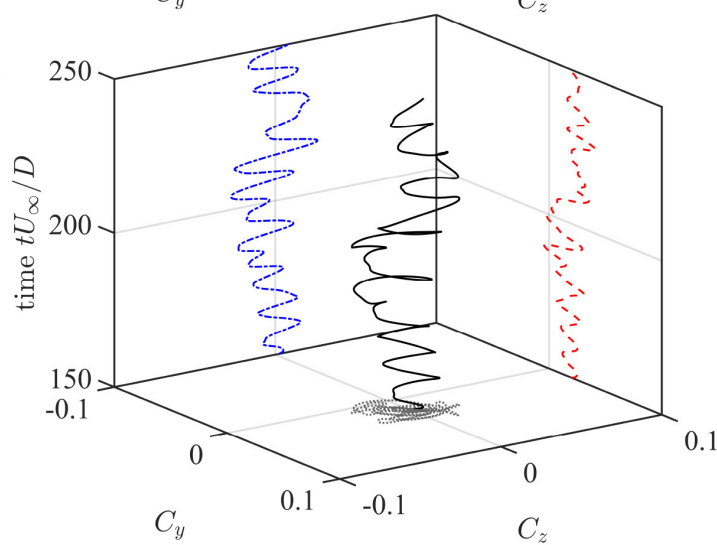

(e)

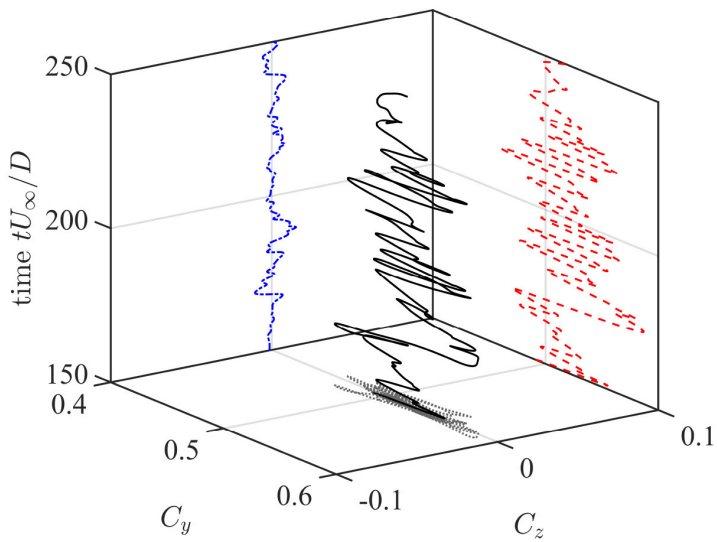

(b)

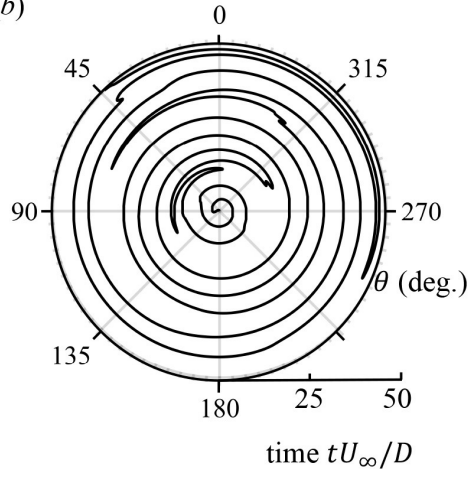

(d)

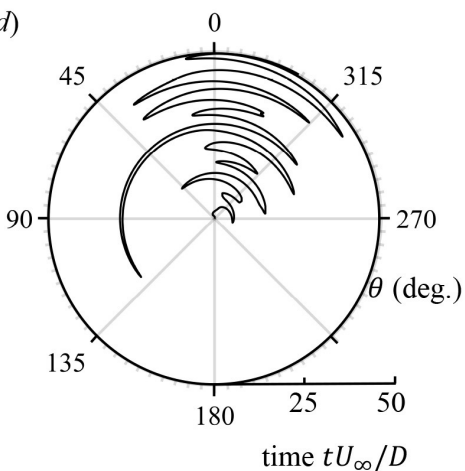

(f)

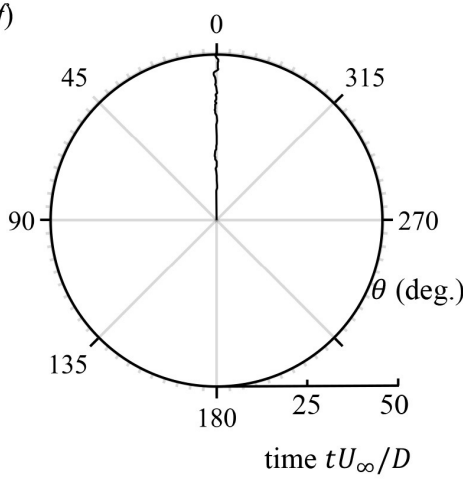

FigURE 16. Instantaneous force coefficients (left column) and temporal evolution of the vortex shedding location (right column) when disk is inclined by $(a-b) \alpha=0^{\circ} ;(c-d) \alpha=1^{\circ} ;(e-f)$ $\alpha=30^{\circ}$ at $R e=300$. The grey dashed line of $C_{y}^{\prime}-C_{z}^{\prime}$ is the time-independent projection of the black solid time trace of $C_{y}-C_{z}$. The blue and red lines are the time traces of $C_{z}$ and $C_{y}$, respectively. The radius of the polar contour is dimensionless time while the polar angle is the azimuthal location of the vortex shedding, which is determined by the orientation of the lateral force (projected onto the disk surface) with respect to the $y$-axis (Shenoy \& Kleinstreuer 2008). The direction of the $y$-axis is represented by the 0 -tick in the polar contour. The rotation of the vortex shedding location appears as an azimuthal expansion from the origin. 
To discover connections between fixed inclined disk and fully rotating freely falling disk (e.g. ZZ or AR states), even though we could easily find counterparts with similar $\chi$ and $R e$ values in the literatures, it is difficult to properly take inertial effects into discussion. Here, the flow pattern of PS state is similar to that of the ZZ mode of the freely falling disk, which shows a planar path with periodic oscillations where $\alpha$ never exceeded $\pi / 2$ (Field et al. 1997; Chrust et al. 2013); the axial vorticity iso-surface also indicated a pair of vortices shedding in opposite orientations (see figure 7 in Chrust et al. 2013). It is more reasonable that the inertia of fixed disk should be infinite, while ZZ mode is extremely suppressed in the high $I^{*}$ area of the parametric diagram (see figure 2 in Auguste et al. 2013). Moreover, Fernandes et al. (2007) reported that translational and rotational degrees of freedom of thin moving bodies can delay the onset of instability when comparing with the critical Reynolds number of Hopf bifurcation for disk placed normal to flow. And this discrepancy would be more significant for inclined disk. We are regretful to draw a conclusion that the SV-ZZ transition of freely falling disk is fundamentally different from the steady state - periodic state transition of fixed one. The transition of the fixed inclined disk is only similar to that in the SV regime, which does not involve significant disk-ambient fluid couplings.

\section{Conclusion and discussion}

Here, the flow around an inclined circular disk is extensively investigated by direct numerical simulations and the transition scenarios in the $R e-\alpha$ parametric space are exhaustively discussed. In the considered $R e-\alpha$ space, five states are observed and denoted as: steady state (SS), periodic state (PS), periodic state with a low frequency modulation (PSL), quasi-periodic state (QP), and chaotic state (CS).

The Reynolds number and inclination angle are the two control parameters studied here, and the effect of the Reynolds number and inclinations on the bifurcation mechanism is discussed. It is evident that a slight break in the perfectly axisymmetric geometry results in a significant change in the flow past disks. The variation of $R e$ gives rise to the bifurcations while $\alpha$ plays a role in modifying the wake patterns, e.g. selecting the plane of reflectional symmetry, changing the frequency of primary vortex shedding and controlling the vortex shedding mechanism. On one hand, Re is always dominant in the transition from the steady to unsteady state and eventually the chaotic state. As shown in figure 5, if viewpoint rolls along with the axis of $R e$, a sequence of bifurcations at $\alpha=0^{\circ}$ is always observed, despite being advanced or delayed at different $\alpha$ values. On the other hand, inclination also plays a dominant role under some conditions, e.g. selecting a fixed and stable plane of reflectional symmetry for the wake over the entire $R e-\alpha$ space (not observed in the blue regions). Inclination breaks the perfect axisymmetric configuration, and therefore it regulates the wake instead of letting it develops arbitrarily. This influence is especially significant for small inclinations where periodic vortex shedding and low frequency pumping motion of recirculation region are stimulated and promoted; the thresholds are advanced. However, at the onset of the RSB mode (a weakly unstable state) Hopf bifurcation is dominant for small inclination angles $\left(\approx 4^{\circ}\right)$ so that the reflectional symmetry could not recover with changing inclination. In the chaotic state, although large inclinations even fails to do so, it also regulates the chaotic motion of the wake. From the perspective of the vortex shedding mechanism, apart from the SS state, for flow behind a slightly inclined circular disk, vortex shedding occurs alternatively on the leading edge and trailing edge. When the inclination angle increases, only the vortex from the trailing edge continues to shed, while the toroidal vortex on the leading edge shows a kind of pumping motion modulated at a low frequency. 
This motion results in a low frequency modulation imposing on the periodic vortex shedding, representing the PSL state. When the inclination angle keeps going up, the upper recirculation bubble remains stably at the disk surfaces and only the trailing edge one sheds. As s result, the periodic state is recovered, even in a harmonic state. Finally, the flow becomes steady when the disk is inclined at a sufficiently large angle, which increases with the increasing $R e$; therefore, the threshold of periodic state - steady state transition is delayed.

Although the correlation between the wake instability of a fixed inclined disk and that of a free falling one is also limited, the present study clarifies the relationship between the fixed body and fluid-body coupled system; the inertial effects which are closely associated with the coupling of the disk and ambient fluid is not relevant for the fixed disk (inclined or not), so these two topics are fundamentally different.

This work is supported by the National Natural Science Foundation of China (Grant nos. 51509152 and 11632011). All the numerical simulations are supported by centre for High Performance Computing, Shanghai Jiao Tong University. The authors are sincerely grateful to Prof. Jun Zhang at New York University for the enlightening discussions. The constructive comments of the reviewers improving the quality of this paper are acknowledged.

\section{REFERENCES}

Auguste, F., Fabre, D. \& Magnaudet, J. 2010 Bifurcations in the wake of a thick circular disk. Theor. Compu. Fluid Dyn. 24 (1), 305-313.

Auguste, F., Magnaudet, J. \& Fabre, D. 2013 Falling styles of disks. J. Fluid Mech. 719, 388-405.

Berger, E., Scholz, D. \& Schumm, M. 1990 Coherent vortex structures in the wake of a sphere and a circular disk at rest and under forced vibrations. J. Fluids Struct. 4 (3), 231-257.

Calvert, J. R. 1967 Experiments on the flow past an inclined disk. J. Fluid Mech. 29 (04), 691-703.

Chrust, M., Bouchet, G. \& Dušek, J. 2010 Parametric study of the transition in the wake of oblate spheroids and flat cylinders. J. Fluid Mech. 665, 199-208.

Chrust, M., Bouchet, G. \& Dušek, J. 2013 Numerical simulation of the dynamics of freely falling discs. Phys. Fluids 25 (4), 044102.

Chrust, M., Dauteuille, C., Bobinski, T., Rokicki, J., Goujon-Durand, S., Wesfreid, J. E., Bouchet, G. \& Dušek, J. 2015 Effect of inclination on the transition scenario in the wake of fixed disks and flat cylinders. J. Fluid Mech. 770, 189-209.

Dickinson, M. H. \& Gotz, K. G. 1993 Unsteady aerodynamic performance of model wings at low reynolds numbers. J. Exp. Biol. 174 (1), 45-64.

Dong, H., Mittal, R. \& NajJar, F. M. 2006 Wake topology and hydrodynamic performance of low-aspect-ratio flapping foils. J. Fluid Mech. 566, 309-343.

Ern, P., Risso, F., Fabre, D. \& Magnaudet, J. 2012 Wake-induced oscillatory paths of bodies freely rising or falling in fluids. Ann. Rev. Fluid Mech. 44, 97-121.

Fabre, D., Auguste, F. \& Magnaudet, J. 2008 Bifurcations and symmetry breaking in the wake of axisymmetric bodies. Phys. Fluids 20, 051702.

Fernandes, P. C., Risso, F., Ern, P. \& Magnaudet, J. 2007 Oscillatory motion and wake instability of freely rising axisymmetric bodies. J. Fluid Mech. 573, 479-502.

Field, S. B., Klaus, M., Moore, M. G. \& Nori, F. 1997 Chaotic dynamics of falling disks. Nature $\mathbf{3 8 8}$ (6639), 252-254.

Horowitz, M. \& Williamson, C. H. K. 2010 The effect of reynolds number on the dynamics and wakes of freely rising and falling spheres. J. Fluid Mech. 651, 251-294.

Jenny, M., DušEK, J. \& Bouchet, G. 2004 Instabilities and transition of a sphere falling or ascending freely in a newtonian fluid. J. Fluid Mech. 508, 201-239. 
Johnson, T. A. \& Patel, V. C. 1999 Flow past a sphere up to a Reynolds number of 300. J. Fluid Mech. 378, 19-70.

Kuo, Y. H. \& Baldwin, L. V. 1967 The formation of elliptical wakes. J. Fluid Mech. 27 (02), $353-360$.

Marshall, D. \& Stanton, T. E. 1931 On the eddy system in the wake of flat circular plates in three dimensional flow. Proceedings of the Royal Society of London. Series A, Containing Papers of a Mathematical and Physical Character 130 (813), 295-301.

Meliga, P., Chomaz, J. M. \& Sipp, D. 2009 Global mode interaction and pattern selection in the wake of a disk: a weakly nonlinear expansion. J. Fluid Mech. 633, 159-189.

Michael, P. 1966 Steady motion of a disk in a viscous fluid. Phys. Fluids 9, 466.

NATARAJAN, R. \& ACRIVOS, A. 1993 The instability of the steady flow past spheres and disks. J. Fluid Mech. 254, 323-344.

OpenFOAM 2009 The Open Source CFD Toolbox, Programmer's Guide, Version 1.6. Boston, MA, USA: OpenCFD Limited.

Rimon, Y. 1969 Numerical solution of the incompressible time-dependent viscous flow past a thin oblate spheroid. Phys. Fluids 12, II-65-75.

Rivet, J. P., Henon, M., Frisch, U. \& D'Humieres, D. 1988 Simulating fully threedimensional external flow by lattice gas methods. Europhys. Letters 7, 231.

Roberts, J. B. 1973 Coherence measurements in an axisymmetric wake. AIAA J. 11, 15691571.

Roos, F. W. \& Willmarth, W. W. 1971 Some experimental results on sphere and disk drag. AIAA J. 9, 285-291.

Shenoy, A. R. \& Kleinstreuer, C. 2008 Flow over a thin circular disk at low to moderate Reynolds numbers. J. Fluid Mech. 605, 253-262.

Shenoy, A. R. \& Kleinstreuer, C. 2010 Influence of aspect ratio on the dynamics of a freely moving circular disk. J. Fluid Mech. 653, 463-487.

TAira, K. \& Colonius, T. 2009 Three-dimensional flows around low-aspect-ratio flat-plate wings at low reynolds numbers. J. Fluid Mech. 623, 187-207.

TiAn, X., Hu, Z., Lu, H. \& YANG, J. $2017 a$ Direct numerical simulations on the flow past an inclined circular disk. J. Fluid Struct. 72, 152-168.

Tian, X., XiaO, L., Zhang, X., Yang, J., TaO, L. \& Yang, D. $2017 b$ Flow around an oscillating circular disk at low to moderate reynolds numbers. J. Fluid Mech. 812, 11191145.

Tomboulides, A. G. \& Orszag, S. A. 2000 Numerical investigation of transitional and weak turbulent flow past a sphere. J. Fluid Mech. 416, 45-73.

Weller, H. G., TABor, G., JAsak, H. \& Fureby, C. 1998 A tensorial approach to computational continuum mechanics using object-oriented techniques. Comput. Phys. 12, 620-631.

Willmarth, W. W., Hawk, N. E. \& Harvey, R. L. 1964 Steady and unsteady motions and wakes of freely falling disks. Phys. Fluids 7 (2), 197-208.

YAng, J., LiU, M., Wu, G., LiU, Q. \& Zhang, X. 2015 Low-frequency characteristics in the wake of a circular disk. Phys. Fluids 27 (6), 064101.

YAng, J., LiU, M., Wu, G., Zhong, W. \& ZhANG, X. 2014a Numerical study on coherent structure behind a circular disk. J. Fluids Struct. 51, 172-188.

YANG, J., TiAn, X. \& LI, X. $2014 b$ Hydrodynamic characteristics of an oscillating circular disk under steady in-plane current conditions. Ocean Eng. 75, 53-63.

Zhong, H. J., Chen, S. Y. \& Lee, C. B. 2011 Experimental study of freely falling thin disks: Transition from planar zigzag to spiral. Phys. Fluids 23 (1), 011702.

Zhong, H. J. \& Lee, C. B. 2012 The wake of falling disks at low Reynolds numbers. Acta Mech. Sin. 28, 1-5.

Zhou, W., Chrust, M. \& DušEk, J. 2017 Path instabilities of oblate spheroids. J. Fluid Mech. 833, 445-468. 